\title{
XV Congresso Brasileiro de Medicina do Esporte Simpósio Luso-Brasileiro de Medicina do Esporte
}

\author{
21 a 23 de junho de 2001 - Hotel Glória - Rio de Janeiro, RJ \\ RESUMOS DOS TEMAS LIVRES
}

\section{ATIVIDADE LOCOMOTORA DE CAMUN- DONGOS APÓS ADMINISTRAÇÃO DE BEBIDA ENERGÉTICA}

Sionaldo E. Ferreira; Isabel M.H. de Quadros; Marco T. de Mello; Maria Lucia O.S. Formigoni.

Depto de Psicobiologia, UNIFESP-EPM, São Paulo - SP.

Fundamentos: relatos populares associados a ausência, quase que total, de trabalhos científicos sobre os efeitos da ingestão de bebidas energéticas (BE), justificam a execução de estudos com o objetivo de quantificar e esclarecer os efeitos produzidos por esses compostos, uma vez que o seu consumo tornou-se popular nos últimos anos. Objetivo: verificar os efeitos da administração aguda de BE na Atividade Locomotora (AL) de camundongos. Metodologia: foram utilizados 80 camundongos com $120 \pm 14$ dias e $43 \pm 3$ gramas. Bebida Energética (Red Bull $\left.{ }^{\circledR}\right)$ em 1, 3 e 5 doses ou água no maior volume; considerando-se uma "dose" de BE a ingestão de 1 lata $(250 \mathrm{ml})$ por uma pessoa de $70 \mathrm{~kg}(3,57 \mathrm{ml} / \mathrm{kg})$. Os experimentos foram realizados em ambiente com temperatura controlada $\left(22 \pm 1{ }^{\circ} \mathrm{C}\right)$; a administração foi por via oral (gavage) e a AL avaliada no Sistema Opto Varimex por 45 minutos. Resultados: observou-se aumento da AL dos animais após a administração de BE em todas as doses (1 dose*: $2320 \pm 707$; 3 doses**: $3012 \pm 669$; 5 doses**: $2945 \pm 967$; controle: 1847 \pm 504) (* p > 0,05; ** p > 0,01; MD \pm DP). Conclusão: a administração de BE aumenta a AL de camundongos e esse efeito é dose / sensibilidade-dependente, visto que com uma dose alguns animais não o apresentaram. Outros experimentos estão sendo realizados para esclarecer quais os componentes da BE provocam este efeito e quais são os efeitos da administração crônica. ApoIo FinanceIro: CNPq, FAPESP \& AFIP.

O2 COMPARAÇÃO DA TAXA METABÓLICA BASAL ENTRE ATLETAS CAMPEÕES COM PREDOMINÂNCIA DO SISTEMA DE FORNECIMENTO DE ENERGIA DE "LONGO E CURTA DURAÇÃO"

Cintia Biehl ${ }^{1-2}$, José N. Guimarães ${ }^{1}$, Renato Alvarenga ${ }^{1}$, Ramón Segura $^{3}$.

Departamento de Biociências da Atividade Física, UFRJ -RJ-Brasil ${ }^{1}$; Instituto do Corpo Integrado à Saúde - Instituto CIS - RJ - Brasil ${ }^{2}$;Universidade de BarcelonaEspanha $;$;e-mail: biehl @ unisys.com.br

Fundamentos: A taxa metabólica basal (TMB) é considerado um dos principais componentes que condicionam o dispêndio de energia total do atleta. Por isto é de interesse a elaboração de classificação da TMB, fundamentada em atletas de alto nível, no estabelecimento de pontos de referência. $\mathrm{O}$ objetivo deste estudo foi o de comparar o TMB entre atletas campeões, alguns com a predominância do sistema de fornecimento de energia de "longa duração" (L-PSFE) (maratona, ciclismo de rota, marcha atlética; $\mathrm{n}=16$; idade $=25,58 \pm$ 2,79 ; estatura $=173 \pm 5,98 \mathrm{~cm}$ ) e outros com predominância do sistema de fornecimento de energia de "curta duração" (CPSFE) (remo; $\mathrm{n}=10$; idade $=23,79 \pm 2,79$ anos; estatura $=$ $189 \pm 3,84 \mathrm{~cm}$ ), atletas masculinos. Metodologia: A data de avaliação do TMB obtido após os Jogos Olímpicos e Mundiais é caracterizada no Calendário Esportivo como um "período pós-competição". Um analisador de gases Mijjnhardt foi usado para calcular o $\dot{\mathrm{VO}}_{2}$ e $\dot{\mathrm{VCO}} 2$. Procedimentos rotineiros foram rigorosamente seguidos na preparação das "condições basais" e durante a medição direta do TMB. A medição do consumo de oxigênio foi realizada em 35 minutos.

\begin{tabular}{c|c|c|c|c|c}
\hline Campeões & $\begin{array}{c}\text { PT } \\
(\mathrm{kg})\end{array}$ & $\begin{array}{c}\mathrm{MCM} \\
(\mathrm{kg})\end{array}$ & $\begin{array}{c}\mathrm{TMB} \\
\mathrm{LO}_{2} \cdot \mathrm{min}^{-1}\end{array}$ & $\begin{array}{c}\text { TMB } \\
\mathrm{mL} \cdot \mathrm{kgPT}^{-1} \cdot \mathrm{min}^{-1}\end{array}$ & $\begin{array}{c}\text { TMB } \\
\mathrm{mL} \cdot \mathrm{kgMCM}^{-1} \cdot \mathrm{min}^{-1}\end{array}$ \\
\hline "L-PSFE" & $64.57 \pm$ & $60.10 \pm$ & $0.24 \pm 0.05$ & $3.65 \pm 0.69$ & $3.92 \pm 0.74$ \\
& 4.1 & 3.78 & & & \\
\hline "C - PSFE" & $89.35+$ & $81.71 \pm$ & $0.33 \pm 0.05$ & $3.67+0.73$ & $4.01 \pm 0.79$ \\
& 5.1 & 3.98 & & & \\
\hline
\end{tabular}

Estes resultados sugerem que os atletas C-PSFE têm mais peso total (PT) e têm mais massa corporal magra (MCM), o que pode sugerir um TMB maior. As percentagens relativas mostraram que os atletas C-PSFE tiveram $27 \%$ mais TMB absoluta $\left(\mathrm{LO}_{2} \cdot \mathrm{min}^{-1}\right)$ que os atletas L-PSFE, contudo não houve diferença significativa na TMB relativa (MCM e PT). Conclusão: Um aumento da TMB em relação a PT e MCM em atletas L-PSFE pode ser resultante da predominância do sistema de energia de "longa duração", desenvolvido durante o treinamento. Apoiado pela FAPERJ e Instituto CIS.

\section{DETERMINAÇÃO DO GASTO ENERGÉTICO DE JOGADORES DE FUTEBOL DURANTE TREI- NAMENTO UTILIZANDO-SE PARÂMETROS ER- GOESPIROMÉTRICOS}

Alessandra Favano; André Pedrinelli; Paulo R.S. Silva; Fábio J. Angelini; Eures S. Facci; Marcelo M. Gondo; Ricardo Galotti; Alberto A. A. Teixeira; Marco M. Amatuzzi

Grupo Integrado de Medicina do Esporte do IOT - HCFMUSP - São Paulo/ SP

Fundamentos: $O$ futebol é um esporte que demanda atividade física combinada e intermitente com graus variados de intensidade realizados continuamente durante uma partida ou treino. Objetivo: O objetivo deste trabalho foi estimar o gasto energético de cada atleta utilizando-se os limiares ventilatórios $1\left(\mathrm{LV}_{1}\right)$ e $2\left(\mathrm{LV}_{2}\right)$ e o consumo de oxigênio de pico ( $\dot{\mathrm{VO}}_{2}$ pico) como marcadores representativos das intensidades utilizadas durante treinamento. Metodologia: Foram avalia- 
dos 18 futebolistas com média de idade de 23,7 $\pm 3,3$; peso $=$ $76,6 \pm 8,1$ que foram submetidos a avaliação cardiorrespiratória e metabólica utilizando-se um analisador de gases computadorizado (Sensor Medics, Vंmax 29c, EUA).O cálculo estimado do gasto energético em Kcal nas intensidades determinadas $\left(\mathrm{LV}_{1}+\mathrm{LV}_{2} / 2=\right.$ moderada; $\mathrm{LV}_{2}=$ intensa; $\mathrm{LV}_{2}+$ $\mathrm{V}_{2}$ máx $/ 2=$ muito intensa) foi realizado pela fórmula do American College of Sports Medicine $\left[\mathrm{Kcal} / \mathrm{min}=\left(\mathrm{n}^{\circ} \mathrm{de}\right.\right.$ MET's x 3,5 x Peso corporal [Kg])/ 200] Resultados: Verificou-se os seguintes parâmetros e resultados:

\begin{tabular}{|c|c|c|c|c|c|}
\hline Intensidades & $\begin{array}{c}\mathrm{VO}_{2} \\
(\mathrm{ml} / \mathrm{Kg} / \mathrm{min})\end{array}$ & $\begin{array}{l}\mathrm{N}^{0} \text { de } \\
\text { MET's }\end{array}$ & $\begin{array}{c}\text { Kcal/ } \\
\text { min }\end{array}$ & $\begin{array}{c}\text { Kcal/ } \\
\text { treino }\end{array}$ & $\begin{array}{c}\text { Tempo/ } \\
\text { Treino(min) }\end{array}$ \\
\hline$\underline{L V}_{1}+\frac{L V_{2}}{2}$ & $36,51 \pm 4,65$ & $\begin{array}{c}10,15 \pm \\
1,28\end{array}$ & $\begin{array}{c}13,64 \pm \\
2,48\end{array}$ & $\begin{array}{c}798,11 \pm \\
145,3\end{array}$ & 58,5 \\
\hline $\mathbf{L V}_{2}$ & $41,13 \pm 4,82$ & $\begin{array}{c}11,43 \pm \\
1,33\end{array}$ & $\begin{array}{c}15,37 \pm \\
2,78\end{array}$ & $\begin{array}{c}345,83 \pm \\
62,51\end{array}$ & 22,5 \\
\hline$\underline{\mathrm{LV}}_{2}+\frac{\mathrm{VO}_{2}}{2}$ pico & $45,8 \pm 4,53$ & $\begin{array}{c}12,71 \pm \\
1,26\end{array}$ & $\begin{array}{c}17,09 \pm \\
2,77\end{array}$ & $\begin{array}{c}153,94 \pm \\
24,86\end{array}$ & 9 \\
\hline
\end{tabular}

Conclusão: Os resultados demonstraram que a ergoespirometria é uma metodologia de grande importância não só para a verificação de marcadores de performance atlética, mas também fornece dados que podem ser canalizados para o controle da necessidade calórica através da quantificação do gasto energético durante treinamento.

\section{EFEITO DE ESTERÓIDES ANABOLIZANTES ANDROGÊNICOS NO GANHO DE MASSA MUS- CULAR EM RATOS SUBMETIDOS AO TREINA- MENTO DE NATAÇÃO}

Rodolfo de P. Vieira, Ana Paula M. M. Lopes, José C. Cogo, Rodrigo A. B. Lopes-Martins

Laboratório de Farmacologia e Experimentação Animal-IP\&D; Laboratório de Fisiologia e Junção Neuromuscular-Serpentário - CEN

Fundamentos: Esteróides anabólizantes androgênicos (EAA) incluem a testosterona e seus diferentes derivados sintéticos. Estas substâncias tem sido empregadas com o objetivo de aumento de massa muscular, mas no entanto, sua eficácia ainda é questionável, e são necessários estudos mais aprofundados sobre o tema. Objetivos: Investigar se o efeito de ganho de massa muscular normalmente observado com o uso de EAAs se deve ao aumento de retenção hídrica ou ao aumento da quantidade de proteína contrátil. Metodologia: Foram utilizadas 36 ratas Wistar, pesando $180 \mathrm{~g} \pm 10$ distribuídos em 6 grupos, treinadas e tratadas com esteróides ou não. $\mathrm{O}$ treinamento físico utilizado foi a natação, durante 45 dias. Os EAAs foram administrados por via intramuscular (5dias/semana; $1 \mathrm{mg} / \mathrm{Kg} /$ dia). O peso corpóreo foi determinado em balança digital Mettler, e o peso dos músculos (úmido e seco) Extensor Digitálico Longo e Tibial Anterior foram determinados em balança analítica Hauss (05 casas decimais). Resultados: Os resultados demonstraram que o Deca-Durabolin foi capaz de aumentar o peso corporal dos animais $(\mathrm{p}<0.05)$ em relação a todos os demais grupos. O Deposteron foi capaz de aumentar o peso corporal dos animais em relação ao grupo controle $(\mathrm{p}<0.05)$, demonstrando porém, uma menor atividade anabólica que o Deca-Durabolin, uma vez que observou-se maior retenção hídrica neste grupo. Conclusão: A hipertrofia muscular é intensificada pelo uso de EAAs, mesmo na ausência de treinamento físico. Contudo esse aumento se deve quase que exclusivamente à retenção hídrica e não ao aumento da quantidade de proteína contrátil.

\section{PERFIL DIETÉTICO E CONSUMO DE SUPLE- MENTOS NUTRICIONAIS DE JOGADORES PRO- FISSIONAIS DE FUTEBOL}

Isabela P Guerra,Eliane S Abreu, Roberto C Burini

Curso Interunidades em Nutrição Humana Aplicada - PRONUT - USP - São Paulo $S P$

Fundamentos: A nutrição, o treinamento e a saúde são aspectos importantes para um bom desempenho no esporte. A demanda energética dos treinamentos e competições requer uma dieta balanceada, particularmente rica em carboidratos. Objetivo: verificar o consumo dietético e o uso de suplementos nutricionais de jogadores de futebol. Metodologia: 66 atletas profissionais de 4 diferentes times de futebol. Os dados foram analisados através do recordatório de 24 horas e de um questionário avaliando o uso ou não de suplementos nutricionais. As dietas foram analisadas (programa Virtual Nutri FSP- USP) obtendo-se valores para energia e macronutrientes. Resultados: ingestão energética média diária $=3918,87$ $\mathrm{Kcal}( \pm 1255,95)$. O consumo de carboidrato $(5,82 \mathrm{~g} \pm 2)$ estava abaixo do consumo mínimo estabelecido de $8 \mathrm{~g} / \mathrm{Kg}$ de peso corporal/dia e, o de proteína $(2,48 \mathrm{~g} \pm 0,73)$ bem acima do limite proposto de 1,4 a 1,7 g/ Kg de peso corporal/dia. Todos os clubes estudados utilizam suplemento como: bebidas hidroeletrolíticas e a base de carboidratos, 2 dos clubes além destes suplementos, utilizavam também cápsulas de aminoácidos e, apenas 1 creatina. Conclusão: os jogadores têm adequado consumo energético diário, devendo aumentar o consumo de carboidratos.

\section{RESPOSTAS ENDÓCRINAS AO EXERCÍCIO MÁXIMO EM SEDENTÁRIOS E PRATICANTES DE CORRIDA}

Daniel A. Rosa, Marco T. de Mello, Sionaldo E. Ferreira, Maria L.O.S. Formigoni.

Depto. de Psicobiologia, UNIFESP, São Paulo, SP.

Fundamentos: A prática do exercício físico acarreta em adaptações nas respostas hormonais ao exercício. No entanto, estas respostas são dependentes do tipo, duração e intensidade de esforço realizado. Objetivo: Verificar as respostas de ACTH e cortisol ao teste de esforço em esteira rolante até a exaustão (Teste Ergoespirométrico - TE) em indivíduos sedentários e corredores. Metodologia: A amostra foi composta por 17 voluntários sedentários (G1) com 25 \pm 4 anos de idade e 15 corredores (mínimo 6 h semanais de corrida) com $27 \pm 4$ anos de idade (G2). As amostras de sangue foram coletadas antes $\left(-15^{\prime}\right)$, após (0’) e durante o período de recuperação do TE 
(30'R,60'R,120'R). Resultados: G2 apresentou maior $\mathrm{V}_{2 \text { máx }}$ do que G1 (64 \pm 7 vs $45 \pm 6 \mathrm{~mL}$.Kg. $\mathrm{min}^{-1}$, respectivamente). Não havia diferenças basais (-15') nos níveis de ACTH (19 \pm 8 vs $22 \pm 8 \mathrm{pg} / \mathrm{mL})$ e cortisol $(13 \pm 3$ vs $12 \pm 4 \mu \mathrm{g} / \mathrm{dL})$ entre os G1 e G2. O TE induziu um aumento tanto para ACTH (0') quanto para o cortisol (30' R) em ambos os grupos ( $\mathrm{p}<0,05)$, que não apresentaram diferenças entre si. A análise da Área Sobre a Curva também não detectou diferenças entre os grupos tanto para as respostas de ACTH quanto para as de cortisol (205 \pm 24 vs $212 \pm 18$ pg.min $/ \mathrm{mL} ; 68 \pm 3$ vs $73 \pm 3 \mu \mathrm{g} . \mathrm{min} / \mathrm{dL}$ ). Conclusão: Estes resultados sugerem não haver diferenças entre sedentários e corredores nas respostas de ACTH e cortisol ao exercício máximo (TE).

\section{ANÁLISE DO PERFIL DE RISCO DA DOEN-} ÇA CARDIOVASCULAR DE PACIENTES ENCAMINHADOS AO AMBULATÓRIO DE ATIVIDADE FÍSICA DO HOSPITAL UNIVERSITÁRIO PEDRO ERNESTO

Adriana Rozentul; Luísa R. de Meirelles; Simone Dino; Vivian Liane M. Pinto; Pedro di Marco da Cruz.

Hospital Universitário Pedro Ernesto - Universidade do Estado do Rio de Janeiro

Objetivo: Analisar a composição corporal e sua associação com os fatores de risco da doença arterial coronariana (DAC). Metodologia: Foram avaliados 106 pacientes, sendo 39 do sexo masculino (37\%), com média de idade de 54,1 anos. 43 eram coronariopatas (41\%), 31 hipertensos (29\%), 7 diabéticos (7\%), 8 diabéticos e hipertensos (7\%), 2 portadores de marcapasso (2\%) e 15 saudáveis (14\%). Os indivíduos foram submetidos as seguintes medidas e avaliações: um questionário de história familiar e da atividade física habitual e avaliação da composição corporal. Resultados: A avaliação dos resultados demonstrou que $81 \%$ dos indivíduos eram sedentários, 50\% com história familiar de DAC e $66 \%$ de hipertensão, $7 \%$ tabagistas e $45 \%$ ex-tabagistas. Dos $65 \%$ que apresentaram uma relação cintura/quadril de risco alto a muito alto, $55 \%$ eram sedentários e dos $30 \%$ obesos, $24 \%$ eram sedentários. A média do peso de gordura foi de $24,4 \mathrm{~kg}$ e em relação ao padrão do percentual de gordura, levando-se em consideração o sexo e a idade, observou-se que $45 \%$ dos homens e $56 \%$ das mulheres estavam acima da média. Conclusão: As medidas da composição corporal relacionadas com a saúde devem ser feitas durante toda a vida. $\mathrm{O}$ alto percentual de indivíduos sedentários e com sobrepeso, demonstra a importância de programas que tenham como meta a promoção da saúde.

\section{ASPECTOS MULTIDICIPLINARES EM PA- CIENTES PÓS INFARTADOS}

Anderson Z. Ulbrich; Neiva Leite

D.E.F - Universidade Federal do Paraná - Curitiba (PR)

Fundamentos: $\mathrm{O}$ sedentarismo combinado à outros fatores de risco, associa-se com a doença arterial coronariana (DAC).
Inversamente, a atividade física regular (AFR) é útil na prevenção da DAC. Objetivo: avaliar os fatores de risco na DAC e benefícios da AFR nestes pacientes. Metodologia: Estudo longitudinal com total de 93 pacientes, 32 do sexo feminino(F); 61 masculino(M); média de idade de 54 e 64 anos. Coletou-se mediante prontuário médico: pressão arterial, colesterol, triglicerídios, HDL, LDL, Glicemia, IMC, RCQ. Foram comparados duas avaliações, acompanhados por seis meses de tratamento. Resultados: Níveis pressóricos arteriais mais baixos, tanto em M quanto $\mathrm{F}(\mathrm{p}<0,05)$, e os níveis de colesterol e triglicerídios nas $\mathrm{F}(\mathrm{p}<0,05)$. A abstenção ao tabagismo foi de $59 \%$ para $\mathrm{M}$ e $58 \%$ para F. Houve redução nos medicamentos. Iniciaram AFR 70\% do $\mathrm{M}$ e $56 \%$ do $\mathrm{F}$ e reabilitação de $5 \%$ M e 7\% F. Não ocorreram mudanças nas variáveis antropométricas comparadas, apesar de praticarem exercícios. A prescrição das AFR's não foi controlada pelo tratamento médico e farmacológico. Conclusão: Sugerimos para melhores resultados que, as AFR's desenvolvidas pelo profissional de Educação Física, deva estar associada com outros profissionais para melhorar o processo de recuperação e reeducação destes pacientes.

\section{ATIVIDADES AQUÁTICAS RECREATIVAS: UM ESTÍMULO ALTERNATIVO PARA AS CRIAN- ÇAS E ADOLESCENTES COM SÍNDROME DE DOWN}

Andrea K. Martins, Ricardo A. Mendes, Maria Isabel T. Ruschel, Neiva Leite, Pedro Paulo E. Vera.

Centro Universitário Positivo - UnicenP, Curitiba, PR

Fundamentos: As atividades aquáticas recreativas (AAR) surgiram como uma nova alternativa para a estimulação de pessoas com Síndrome de Down (SD). Objetivos: Verificar as funções psicomotoras, afetivas-sociais e a autonomia das atividades da vida diária (AVDs) em crianças e adolescentes com SD, durante o estímulo das AAR. Metodologia: Estudo longitudinal descritivo, com 19 sujeitos com SD, entre 07 e 16 anos, divididos em: 9 no grupo experimental (GE) e 10 no controle (GC). Utilizou-se o teste KTK de Schilling \& Kiphard (1974) para avaliação da psicomotricidade em 4 subtestes. Foram preenchidos questionários e fichas de observação com os responsáveis acompanhando a afetividade, a sociabilização e a autonomia das AVDs. As AAR foram desenvolvidas no GE, 2 vezes por semana, com duração de 50 minutos, em um período de 4 meses, com incentivo à autonomia das AVDs. Os testes foram aplicados na primeira avaliação $\left(1^{\mathrm{a}}\right)$ e na reavaliação após 4 meses de AAR $\left(2^{\mathrm{a}}\right)$. Resultados: O GE iniciou com índice KTK $=179,11 \pm 53,12\left(1^{\mathrm{a}}\right)$ e modifi-

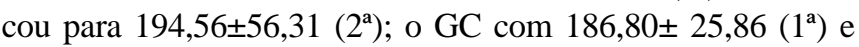
$189,80 \pm 24,04\left(2^{\mathrm{a}}\right)$. No GE houve melhora no comportamento afetivo-social e na autonomia das AVDs em todos alunos. Conclusão: Indivíduos com SD estimulados, desenvolvem-se mais e possuem maior possibilidade de inclusão na sociedade atual. As AAR podem ser uma alternativa para esta população. 


\section{BRONCOESPASMO INDUZIDO POR EXERCÍ-} CIO

Andréa L.G. Teldeschi, Clemax C. Sant'Anna

UFRJ, Universidade Gama Filho

Fundamentos: $\mathrm{O}$ broncoespasmo induzido por exercício(BIE) representa uma condição de hiperresponsividade brônquica. Objetivo: Determinar a prevalência do BIE através de provas de função pulmonar e teste de broncoprovocação por exercício(TBPE) com esteira ergométrica, e correlacionar com as características clínicas e funcionais dos 135 escolares estudados. Metodologia: Estudou-se uma população de asmáticos de 7 a 15 anos, diagnosticados por questionário ATS-DLD78-C, modificado pela autora para diagnóstico de asma em crianças e com relato de broncoespasmo(BE) nos últimos 12 meses, utilizando-se espirógrafo AM 4000 PC da Anamed, Peak Flow Meter Wright, esteira ergométrica ECOFIX EG 700x. Resultados: A prevalência do BIE foi de 57\%(77 entre 135). Dos 135 escolares, 77(57\%) não apresentavam insuficiência ventilatória(IV), 29(22\%) apresentavam IV leve, $23(17 \%)$ IV moderada e $6(4 \%)$ IV moderada a grave. Das variáveis clínicas analisadas, tiveram forte correlação com BIE: relato de tosse, cansaço com chiado, início precoce da asma, evolução inalterada ou com piora nos últimos 12 meses, asma grave e exercício ou alterações climáticas como desencadeante do BE. Durante o TBPE, apenas 32 escolares, dos 77 com BIE, apresentaram concordância entre história, sinais clínicos de BIE e diagnóstico laboratorial do BIE. Conclusão: $\mathrm{O}$ diagnóstico correto do BIE só é possível com TBPE. apoio CNPq, processo: 352009/96

\section{EXERCÍCIO FÍSICO REGULAR MELHORA A CAPACIDADE FUNCIONAL E O SISTEMA IMU- NOLÓGICO DE PACIENTES COM HIV/AIDS}

Gladys C. F. Lavado, Wellington A. M. Silva, Carlos E. Negrão. Escola de Educação Física e Esporte e Instituto do Coração (InCor) HC-FMUSP, São Paulo, Brasil

Fundamentos: A AIDS é uma doença multifatorial, complexa e de prognóstico variável. Portanto, a busca de condutas terapêuticas para melhorar a qualidade de vida de pacientes portadores de HIV/AIDS é um assunto que suscita muito interesse. Objetivo: Estudar os efeitos de um programa de exercício físico regular nas respostas de pressão arterial (PA), freqüência cardíaca (FC) e consumo de oxigênio de pico $\left(\dot{\mathrm{VO}}_{2 \text { pico }}\right)$, e no funcionamento do sistema imunológico, em pacientes portadores de HIV/AIDS. Metodologia: 32 indivíduos foram subdivididos em pacientes soropositivos sedentários (HIVPS, $\mathrm{n}=7$ ) e treinados (HIVPT, $\mathrm{n}=9$ ), e indivíduos soronegativos sedentários (HIVNS, $\mathrm{n}=8$ ) e treinados (HIVNT, $\mathrm{n}=8$ ). O programa de exercício físico foi realizado por 12 semanas, duas vezes por semana. Foi realizado um teste de esforço máximo antes e após o período experimental. O sistema imunológico foi avaliado através da contagem de células CD4. Resultados: Ao contrário do que foi observado no HIVPS, no HIVPT o $\dot{V O}_{2 \text { pico }}\left(37 \pm 2\right.$ vs $\left.32 \pm 2 \mathrm{ml} \cdot \mathrm{kg}^{-1} \cdot \mathrm{min}^{-1}\right)$ e o tempo de teste de esforço máximo aumentaram significativamente e semelhantemente ao HIVNT. Houve aumento significativo da contagem de células CD4 no HIVPT, mas não em pacientes HIVPS. Conclusões: Exercício físico regular aumenta a capacidade funcional e melhora o funcionamento do sistema imunológico de pacientes portadores de HIV/AIDS, podendo ser recomendado como um procedimento coadjuvante no tratamento desses pacientes.

12 PREVALÊNCIA DE SINTOMAS RESPIRATÓRIOS AOS EXERCÍCIOS EM 2941 ESCOLARES DE DUAS REGIÕES ADMINISTRATIVAS DO RIO DE JANEIRO

Clemax C. Sant'Anna, Vera T. Aires, Andréa L.G. Teldeschi

Universidade Gama Filho, Universidade Federal do Rio de Janeiro.

Fundamentos: $\mathrm{O}$ exercício é reconhecidamente um fator desencadeante de broncoespasmo na faixa etária pediátrica. Objetivo: Determinar a prevalência do relato de tosse, cansaço, chiado, dor no peito ou apenas falta de ar após jogos ou exercícios e verificar se há diferença entre os escolares das redes pública e particular. Metodologia: Realizou-se um inquérito transversal em 2941 escolares de 6 a 14 anos, dos quais 1591 eram da rede municipal(RM), 1350 da particular(RP) e 638 eram asmáticos(354 da RM e 284 da RP). Resultados: Houve referência a tosse, cansaço, chiado, dor no peito ou apenas falta de ar após jogos ou exercícios em 684 escolares(23,3\%). A comparação entre os escolares das duas redes mostrou maior prevalência destes sintomas entre os da RM, $26,3 \%$ contra $19,6 \%$ da RP. Dentre os asmáticos, também foi maior a prevalência na RM, 74\% contra $63 \%$ da RP. Conclusão: A prevalência de sintomas aos exercícios físicos é mais elevada na rede municipal sendo acentuada entre os asmáticos de ambas as redes.

\section{VOLUME DE TREINAMENTO E LESÕES OS- TEOMUSCULARES EM CORREDORES}

Valério H. Dezan; Thiago Sarraf; Neiva Leite; Ricardo A. Mendes Departamento de Educação Física - UFPR - Curitiba - PR

Fundamentos: $O$ volume de treinamento excessivo está associado a maior incidência de lesões esportivas em maratonistas. Objetivo: Verificar se existe relação entre o volume semanal de treino com a frequiência das lesões (\%), idade, local lesionado e IMC. Metodologia: Estudo transversal, com 53 homens, na faixa etária de 20 a 40 anos, que preencheram questionário enviado pelo correio, logo após a maratona de Porto Alegre, em 1994. Foram divididos conforme o volume de treino em quilômetros por semana $(\mathrm{km} / \mathrm{sem})$ e relacionados com a indicação de lesões e a localização da mesma. Resultados: 


\begin{tabular}{lcccccc}
\hline Dados $\backslash \mathrm{km} / \mathrm{sem}$ & -12 & $12-24$ & $25-49$ & $50-64$ & $65-95$ & +95 \\
\hline$n$ & 4 & 4 & 10 & 12 & 14 & 9 \\
idade-média/dp & $31 \pm 7,3$ & $31 \pm 5,4$ & $31 \pm 4,3$ & $33 \pm 4,7$ & $32 \pm 3,4$ & $34 \pm 3,7$ \\
IMC- média & 23,19 & 24,26 & 23,82 & 22,92 & 22,25 & 21,34 \\
$\%$ de lesões & 100 & 75 & 70 & 75 & 50 & 100 \\
local + freq. & quadril & Joelho & joelho & joelho & Joelho & calcan. \\
\hline
\end{tabular}

Conclusão: Os volumes de treino inferiores e superiores em $\mathrm{km} / \mathrm{sem}$ demonstraram uma maior ocorrência de lesões, talvez associado a um nível de condicionamento inadequado e a um excesso de treino, respectivamente. Como esperado, o IMC diminuiu com o aumento do trabalho aeróbio semanal e as lesões foram mais freqüentes em membros inferiores. Sugerimos que outras questões devam ser avaliadas na ocorrência das lesões esportivas, pelo aspecto multifatorial envolvido nestes eventos.

\section{COMPROMETIMENTO ARTICULAR EM IN- DIVÍDUOS HEMOFÍLICOS}

Patricia S. Zavelinski, Neiva Leite

Depto. Educação Física, Universidade Federal do Paraná.

Fundamentos: Hemofilia é um distúrbio hereditário da coagulação, caracterizado por hemorragias musculares e articulares, causando hematomas, hemartroses, atrofias musculares e dor intensa. Objetivos: Analisar o comprometimento articular, verificando quais articulações e quais lados mais freqüentemente acometidos. Metodologia: Foram avaliados prontuários e fichas médicas de 125 pacientes hemofílicos atendidos na Hemepar e na Casa do Hemofílico desde 1998 à 2000, para identificação de qual lado ou articulação mais freqüentemente afetada ou acometida. Resultados: Dos 125 pacientes analisados, mais de 50\% tem duas ou mais articulações atingidas. Verificou-se uma diferença significativa em relação à membros inferiores(M.I.). A articulação mais atingida foi o joelho com $61,6 \%$, seguida pelo cotovelo com $31,2 \%$ e pelo tornozelo com $30,4 \%$. As articulações de M.I. foram as mais atingidas provavelmente por suportarem maiores pressões durante o movimento na utilização diária. Não houve diferença entre o acometimento do lado direito em relação ao esquerdo. Conclusão: A importância clínica de avaliar as articulações mais acometidas está relacionada a possibilidade da prevenção. Os profissionais de Educação Física devem participar deste processo, em conjunto com outros profissionais, porque a prevenção de futuras lesões em pacientes hemofílicos deve ser iniciada na escola. O professor deve conhecer a patologia, suas complicações e o seu manejo proporcionando um trabalho de força muscular, evitando possíveis transtornos futuros.

\section{HISTÓRICO DE TREINAMENTO E LESÕES OSTEOMUSCULARES EM CORREDORES}

Thiago A. Sarraf; Valério Dezan; Neiva Leite; Ricardo A. Mendes Departamento de Educação Física - UFPR - Curitiba - PR

Fundamentos: As lesões esportivas em corredores podem estar relacionadas com o volume semanal de treinamento.

Rev Bras Med Esporte - Vol. 7, № 3 - Mai/ J un, 2001
Objetivo: Analisar a relação entre volume semanal de treinamento, IMC e idade com a incidência de lesões e locais lesionados em maratonistas. Metodologia: Estudo transversal, com 46 homens, na faixa etária de 20 a 68 anos, que participaram da maratona de Curitiba de 1997. Foram divididos de acordo com o volume de treino em quilômetros por semana $(\mathrm{km} / \mathrm{sem})$ e a presença e localização de lesões. Resultados: A articulação mais lesionada foi o joelho em $26 \%$. A tabela abaixo registra os principais resultados:

\begin{tabular}{lccccc}
\hline Dados $\backslash \mathrm{km} / \mathrm{sem}$ & $12-24$ & $25-49$ & $50-64$ & $65-95$ & +95 \\
\hline$n$ & 4 & 2 & 8 & 14 & 18 \\
Idade-média/dp & $29 \pm 6,4$ & $48 \pm 9,2$ & $48 \pm 14$ & $44 \pm 9,7$ & $37 \pm 7,6$ \\
IMC- média & 22,58 & 24,5 & 22,61 & 21,84 & 21,23 \\
$\mathrm{~N}^{\text {o de lesionados }}$ & 2 & 1 & 7 & 11 & 10 \\
$\mathrm{~N}^{\text {o de lesões }}$ & 03 & 01 & 14 & 25 & 22 \\
\hline
\end{tabular}

Conclusão: Os atletas lesionados com volume de treino acima de $50 \mathrm{~km} / \mathrm{sem}$ apresentaram mais de uma lesão. O IMC se manteve no padrão de normalidade independente do volume semanal e da faixa etária. Sugerimos, pela natureza multifatorial das lesões, que outros trabalhos avaliem os aspectos psicológicos, as técnicas de corrida, os métodos de treino, os fatores genéticos e a participação em outros esportes.

16 RECONSTRUÇÃO DO LIGAMENTO CRUZADO ANTERIOR COM OS TENDÕES SEMITENDÍNEO E GRÁCILES QUÁDRUPLO (STGQ) FIXADO COM TRANSFIX E PARAFUSO BIODEGRADÁVEL (BIOSCREW) POR VIDEOARTROSCOPIA

Cláudio Gholmia, Daphnis G. Souza.

Depto Medicina Esportiva, Uniort Unidade de Ortopedia e Traumatologia, São Paulo, $S P$.

Fundamentos: Utilização de uma nova opção de enxerto, com uma excelente fixação no fêmur e na tíbia, nas reconstruções do LCA por videoartroscopia. Objetivo: A proposta desse estudo é demonstrar as vantagens e desvantagens como também os excelentes resultados como opção de escolha desse tipo de enxerto para as reconstruções do LCA. Metodologia: Estudo de corte de 100 pacientes operados, sendo avaliados um total de 91 pacientes ( 83 homens; 08 mulheres; idade mínima de 17 anos e máxima de 58 anos, média de 32,4 anos) acompanhados por 18 meses. Resultados: Foi utilizado os critérios de avaliação da Internacional Knee Documentation Committe (IKDC) normal e subnormal 90\% (normal 64 casos e subnormal 18 casos); anormal 4,3\% (4 casos); muito anormal 5,4\% (5 casos). Conclusão: $O$ uso desta opção de enxerto permite afirmar que é uma técnica rápida e segura. A fixação femoral e tibial é rígida, possui poucas complicações, diminui a morbidade, incisão estética e pequena, promove um retorno rápido às atividades e permite o uso do protocolo de reabilitação não agressivo. 


\section{TENORRAFIA PERCUTÂNEA E REABILITA- ÇÃO DO TENDÃO DE AQUILES}

Jaime Milheiro, Paulo Amado, Espergueira Mendes

Unidade de Traumatologia Desportiva do Serviço de Ortopedia e Traumatologia do Hospital São Sebastião, Portugal

Fundamentos: Os dois grandes princípios de tratamento das roturas do tendão de Aquiles, ortopédico e cirúrgico são controversos: o $1^{\circ}$ com maior risco de roturas iterativas e o $2^{\circ}$ com risco séptico e de necrose cutânea. Ma e Griffith (1977), Nada (1985) e Aldam (1989) surgeriram a sutura percutânea como método alternativo combinando vantagens e minimizando complicações resultando numa melhor reabilitação. Objetivo: Demonstrar a eficácia da tenorrafia percutânea no tratamento da rotura do tendão de aquiles. Metodologia: De 1997 a 2000 foram operados 12 doentes (9 homens; 3 mulheres; idades entre os 17 e 47). O tempo médio entre a lesão e a cirurgia foi de 7 dias. Usou-se a técnica de Ma e Griffith modificada, obedecendo a um protocolo de imobilização e recuperação comuns. O pós-operatório compreendeu protecção com bota funcional 4 a 6 semanas e carga imediata acompanhado de tratamento fisiátrico. Resultados: Ausência de roturas iterativas e necroses cutâneas. Avaliaram-se os parâmetros dor, mobilidade articular final, força e atrofia muscular da perna, capacidade de se colocar em bicos dos pés, salto unipodal e avaliação funcional com a tabela de McComis modificada, com Ecografia (RM em 2 casos) pré e pós-operatória. Após reabilitação todos recuperaram a actividade profissional e desportiva. Conclusão: A sutura percutânea é um dos métodos de eleição no tratamento das rotura aguda do tendão de Aquiles não pretendendo substituir os anteriores pois tem indicações precisas e limites. Pensamos ser o mais vantajoso sendo na actualidade a técnica de eleição no tratamento cirúrgico nas roturas do tendão de Aquiles.

\section{TRATAMENTO CIRÚRGICO DA INSTABILI- DADE ANTERIOR DO OMBRO POR VIA AR- TROSCÓPICA}

R. Catena; W. Castropil; B. Schor

Instituto Vita de Medicina Esportiva

Objetivo: Analisamos 38 pacientes submetidos ao tratamento cirúrgico da instabilidade anterior do ombro por via artroscópica. Metodologia: Todos os pacientes apresentavam instabilidade do ombro, sendo 30 de origem traumática e 8 de origem atraumática. Do total, a reparação do lábio por reinserção com o uso de âncora foi realizado em 31 pacientes e a termocapsuloplastia foi realizada em todos (6 com o uso do laser Ho:YAG e o restante com o uso de radio-freqüência). Todos os pacientes foram mantidos com o uso de imobilização tipo Velpeau por 3 semanas e seguiram o mesmo protocolo de reabilitação. Os pacientes, 34 do sexo masculino e 4 do sexo feminino foram estudados após um tempo de seguimento médio de 16 meses (6 a 30 meses), através de anamnese e exame físico. Resultados: Em 35 pacientes $(92,1 \%)$ não hou- ve recidiva da instabilidade com limitação da amplitude de rotação externa média de 3 graus. Em 15 pacientes realizamos avaliação isocinética da musculatura do ombro que mostrou ausência de déficit significante em relação ao lado contralateral. Como complicações, obtivemos recidiva da instabilidade em 3 pacientes $(7,9 \%)$ e 2 pacientes $(5,3 \%)$ apresentam soltura das âncoras de fixação com impacto na cabeça umeral. Conclusão: Concluímos que, na casuística estudada, o método mostrou-se eficaz na estabilização do ombro, com pouca limitação da amplitude articular. As complicações podem se minimizadas com o uso preferencial de âncoras de material bioabsorvível.

\section{A APTIDÃo FíSICA E A PRÁTICA DE ATIVI- DADE FÍSICA DE LAZER AFETAM A PRECISÃO NOS RELATOS DE PESO E ALTURA}

Geraldo A. Maranhão Neto, Marcos D. Polito, Vitor A. Lira Corpo \& Água Sports Center, Petrópolis - RJ.

Fundamentos: Estudos epidemiológicos fazem uso freqüente de variáveis auto-reportadas, como peso e altura. Objetivos: Verificar a influência da aptidão física e prática de atividade física de lazer sobre a precisão no relato de peso, altura e IMC. Metodologia: Avaliou-se o $\mathrm{V}_{2}$ max (Åstrand-Rhyming, 1954), a flexibilidade geral (FG), com o Flexiteste (Araújo, 1986) adaptado, o percentual de gordura (\%g), a circunferência abdominal (CA), o peso e a altura relatados e medidos em 200 mulheres $(34 \pm 11$ anos $)$ e 128 homens $(33 \pm 11$ anos [média $\pm \mathrm{dp}]$ ), que procuraram a prática supervisionada de atividades físicas. Considerou-se sedentários os que apresentavam, nos últimos três meses, freqüência semanal de atividades físicas de lazer $(\mathrm{FSA})<1$, como pouco ativos $(1 \leq$ FSA $<3$ ) e como fisicamente ativos $(F S A \geq 3)$. As diferenças entre o relatado e medido para peso (DP), altura (DA) e IMC (DIMC) foram, então, analisadas. Resultados: Homens e mulheres superestimaram a altura em $0,44 \pm 1,7$ e 0,29 \pm $2,1 \mathrm{~cm}$, respectivamente, porém as mulheres subestimaram o peso em $0,05 \pm 1,5 \mathrm{~kg}$ e os homens o superestimaram em $0,3 \pm$ $2,9 \mathrm{~kg}$. A regressão múltipla stepwise forward mostrou correlações da DP com CA, $\dot{V O}_{2}$ e FSA $\left(r=0,28, r^{2}{ }_{a}=0,07, p=\right.$ $0,0007)$ e de DIMC com CA, FSA, idade, $\dot{\mathrm{VO}}_{2}$ e FG $(\mathrm{r}=0,33$, $\left.\mathrm{r}^{2}{ }_{\mathrm{a}}=0,09, \mathrm{p}=0,0004\right)$, nas mulheres e, de DP com CA, FG, altura e\%g $\left(r=0,38, r^{2}{ }_{a}=0,12, p=0,0006\right)$ e DIMC com CA, FG e $\dot{V O}_{2}\left(r=0,33, r^{2}=0,09, p=0,002\right)$, nos homens. Conclusão: Parece haver influência da aptidão física e da prática de atividade física na precisão dos relatos, possivelmente, por indivíduos mais aptos e ativos controlarem o peso e o IMC com maior facilidade.

\section{ATIVIDADE FÍSICA E QUALIDADE DE VIDA ENTRE IDOSOS COM OSTEOPOROSE: UMA REVISÃO}

Jani C. B. de Aragão, Estélio H. M. Dantas.

PROCIMH, Universidade Castelo Branco, Rio de Janeiro, RJ. 
Fundamentos: $\mathrm{O}$ declínio da resistência musculoesquelética acarretado pela osteopenia e pela deterioração microarquitetural (osteoporose) aumenta o risco de quedas e fraturas em idosos, prejudicando sua autonomia e qualidade de vida. Objetivos: Examinar e avaliar a relação entre atividade física e qualidade de vida em idosos portadores de osteoporose. Metodologia: Uma revisão bibliográfica selecionou 96 documentos, dentre os quais uma meta-análise (KELLEY e col., 2000), três revisões (GREGG e col., 2000, LAYNE e col., 1999 e wOLFF e col., 1999) e dois posicionamentos (ACSM, 1995 e FORWOOD e col. 2000). Estudos com grupos aleatórios também foram incluídos. Resultados: Dentre estudos selecionados 48,95\% defendem a atuação em qualquer atividade física (atividade aquática, 4,16\%; caminhada, 6,25\%; desportos, 6,25\%; corrida, $12,5 \%$, fitness físico, 19,79\%). Atividades com sobrecarga são as mais citadas $(41,6 \%)$ como fomentadoras da melhoria da osteoporose. Em três artigos a osteoporose é citada como doença iniciada na infância pela falta de atividade física. A qualidade de vida é citada em todos os artigos como produto final dos benefícios causados pela atividade física. Conclusão: A atividade física proporciona melhora na densidade óssea, sobretudo se houver freqüência, intensidade e duração elevadas, utilizando-se uma sobrecarga. Além do mais, pelo seu potencial o exercício melhora a saúde e o bem estar, elevando a qualidade de vida.

\section{ATIVIDADE FÍSICA HABITUAL E DENSIDA- DE MINERAL ÓSSEA EM HOMENS ADULTOS E IDOSOS}

Alex A. Florindo; Maria do Rosario D.O. Latorre; Patricia C. Jaime; Tomoe Tanaka; Cristiano A.F. Zerbini

Depto de Epidemiologia da Faculdade de Saúde Pública da USP; Hospital Heliópolis/São Paulo

Objetivo: Verificar a correlação entre a atividade física habitual (AFH) ao longo da vida e a densidade mineral óssea (DMO) em homens adultos e idosos. Metodologia: Foram analisados 326 homens com idade igual ou superior a 50 anos, voluntários da grande São Paulo. Os dados de DMO foram coletados através de densitometria óssea das regiões de corpo total, colo do fêmur, triângulo de ward, trocanter e coluna lombar. Os dados de AFH foram coletados através de questionários, com questões referentes aos exercícios físicos, atividades físicas de lazer e atividades físicas ocupacionais nos períodos de 10 a 20 anos de idade, de 21 a 30 anos, de 31 a 50 anos e dos últimos 12 meses e atividades físicas de locomoção dos últimos 12 meses. A relação entre as variáveis foi analisada através de regressão linear múltipla. Resultados: $\mathrm{Na}$ análise multivariada, depois do ajuste por idade e IMC, as variáveis independentes que melhor explicaram a variação da DMO em todos os locais analisados foram os exercícios físicos e atividades físicas de lazer praticados no período de 10 a 20 anos e as atividades físicas de locomoção dos últimos 12 meses para as regiões do corpo total, triângulo de ward, tro- canter e coluna lombar e as atividades físicas ocupacionais dos últimos 12 meses para o colo do fêmur. Conclusão: Os exercícios físicos e atividades físicas de lazer do período de 10 a 20 anos e as atividades físicas de locomoção contribuiram para preservação da DMO em homens adultos e idosos. Apoio: FAPESP proc. 98/02770-6

\section{AVALIAÇÃO DO TREINAMENTO FÍSICO DO AERONAUTA-AEROFIT QUANTO AOS ASPEC- TOS DE COMPREENSÃO, AGRADABILIDADE, ADEQUABILIDADE E VIABILIDADE}

Leônidas P. Bomfim, Estélio H. M. Dantas.

Universidade Castelo Branco, Rio de Janeiro, RJ

Fundamentos: Estudos recentes revelam que a fadiga apresenta-se como um risco potencial para a segurança de vôo, tendo sido apontada como uma das principais causas de acidentes aéreos. Entretanto, observa-se ainda um reduzido número de pesquisas efetuadas para elaboração de programas de condicionamento físico com o objetivo de aumentar a tolerância à fadiga pelos tripulantes, (ASRS, 1994). Objetivo: Avaliar o treinamento físico para aeronautas - AEROFIT quanto aos aspectos de compreensão, agradabilidade, adequabilidade e viabilidade visando ao aperfeiçoamento do programa. Metodologia: Aplicação de questionário de avaliação em 32 aeronavegantes (12 pilotos, 10 mecânicos de vôo e 10 comissários; idade $33 \pm 5$ anos) após experimento durante o projeto piloto. Foi apreciado separadamente cada subprograma do AEROFIT, que é composto de um treinamento aeróbico (FITrobic), localizado (FITloc) e de flexibilidade (FITflex). Resultados: Compreensão e agradabilidade: excelente $=20 \%$, boa: $40 \%$, normal: $26 \%$, satisfatória: $14 \%$, deficiente: $0 \%$; adequabilidade: excelente: $19 \%$, boa: $42 \%$, normal: $28 \%$, satisfatória: $7 \%$, deficiente: $4 \%$; viabilidade: excelente: $11 \%$, boa: $20 \%$, normal: $32 \%$, satisfatória: $23 \%$, deficiente: $14 \%$. Conclusões: As análises dos questionários nos levaram a concluir que o AEROFIT é um programa de exercícios de fácil compreensão, agradável e adequado, contudo, necessita de alguns ajustes quanto ao aspecto da viabilidade de algumas partes de seus subprogramas.

\section{ESTUDO DO SOMATÓTIPO DAS SELEÇÕES BRASILEIRA, ARGENTINA, PARAGUAIA, URU- GUAIA E CHILENA, DA CATEGORIA JÚNIORES DE HANDEBOL MASCULINO-2000}

Mônica Sêncio Paes Langoski, Fábio Roberto Langoski

Universidade Castelo Branco - Rio de Janeiro.

Fundamentos: Somatotipo é a representação numérica de uma escala que define se a pessoa apresenta um nível elevado de endomorfia, de mesomorfia e de ectomorfia. Os atletas são nitidamente diferentes da população comum, indicando a necessidade de uma predisposição natural para o sucesso, uma vez que a relação entre estrutura e a função é fator importante na performance de um determinado desporto. Objetivo: O 
presente estudo teve como objetivo determinar o somatótipo das Seleções Brasileira, Argentina, Paraguaia, Uruguaia e Chilena de Handebol Masculino na categoria Júnior-2000 e também analisar de forma comparativa as diferenças somatotipológicas entre as Seleções. Metodologia: As avaliações foram realizadas em 74 atletas, sendo 16 do Brasil, 16 da Argentina, 16 do Paraguai, 16 do Uruguai e 10 do Chile. Para o estudo utilizou-se a metodologia do somatotipo antropométrico proposto por HEATH \& CARTER. Resultados: Os valores médios de somatótipos encontrados foram: Brasil: 3,7 3,33 - 2,78, Argentina: 4,59 - 4,35 - 1,83, Paraguai:4,46 3,02 - 2,07, Uruguai: 4,34 - 4,58 - 2,26, Chile: 4,82 - 4,01 2,38). Conclusão: Concluímos que os atletas das Seleções do Brasil, Argentina, Uuruguai se apresentam como Endomorfo Mesomorfo e os a Seleção do Paraguai e Chile como Endo Mesomórficos.

\section{IDADE DA MENARCA EM JOGADORAS DE VOLEIBOL \\ Cláudio O.V. Lima e Paulo R.S. Amorim \\ Depto. de Educação Física, Universidade Federal de Viçosa, MG}

Fundamentos: A menarca é um acontecimento singular na vida da jovem e as transformações que se desencadeam nesse período afetam os resultados da prática desportiva. Objetivo: Verificar a associação entre a idade de surgimento da menarca (IM) a fatores tais como prática de atividade física prémenarca (AFPM), nível social (NS), formação dos pais (FP) e ordem de nascimento (ON). Metodologia: Amostra foi composta por 175 meninas com faixa etária de 12,4 a 19,9 anos $(\mathrm{X}=15,2 \pm 1,6)$. Os dados foram coletados através de questionário. Utilizou-se a correlação de Spearman por postos na análise do NS, da FP e da AFPM. Em função das múltiplas possibilidades e superposições da ON utilizou-se a ANOVA One Way.para analisar este fator. Resultados: A média de IM foi de $12,0 \pm 1,1$ anos. A FP e o NS não se correlacionaram significativamente $(\mathrm{p}>0,05)$ com a IM. A ON não apresentou diferenças estatisticamente significativas $(p>0,05)$ em relação a IM. A AFPM correlacionou-se significativamente $(\mathrm{p}<0,001)$ com a IM. Conclusão: Os valores da IM desse estudo foram inferiores a outros valores verificados na literatura. FP, NS, e ON não estavam associados a IM. A AFPM apresentou-se como um fator influenciador no retardamento da menarca. Sugerimos que futuros estudos considerem outros fatores que possam influenciar a IM, tais como tendência secular, nível cultural, e que quantifiquem as intensidades do treinamento do período pré-puberal.

\section{INCIDÊNCIA DE PATOLOGIAS EM MMII, DE JOGADORES DE FUTEBOL AMADOR E PROFIS- SIONAL DA $1^{\text {a }}$ DIVISÃO DO ESTADO DO RIO DE JANEIRO, COM BASE NA AVALIAÇÃO DE FOR- ÇA ISOCINÉTICA}

Marceli O. Nascimento, Alexandre H. Pimentel, Sônia M. J. Jesus. Depto. de Futebol Amador, Projeto SOMA, C. R. Flamengo, RJ
Fundamentos: No treinamento de futebolistas são enfatizados os exercícios que fortalecem a musculatura típica da performance, sobretudo extensores do joelho. Com isso a musculatura antagonista (flexores) tem seu significado subestimado, aumentando assim, a probabilidade de lesões musculares, articulares e ligamentares. Objetivo: Associar a incidência de patologias aos desequilíbrios das musculaturas ext. e flex. do joelho. Metodologia: Foram avaliados 93 atletas do sexo masculino, jogadores de futebol, em aparelho isocinético. O teste consistiu em: 5 repetições de ext. e flex. de joelho na velocidade angular de $60 \%$ s, nos MMII. Os relatos das patologias encontradas, foram obtidos dos diários de ocorrências médicas, na temporada 2000/2001. Resultados: 62\% da amostra total apresentaram desequilíbrios musculares entre ext. e flex. de joelho, sendo a ext. $40 \%$ maior que a flex.. Somente a partir destes valores foram encontradas as patologias: Mialgia da musculatura posterior da coxa 22,41\%; Microruptura Muscular 5,17\% e ainda 3,45\% entorses de joelho. Conclusão: Todas as patologias relatadas foram encontradas nos atletas com desequilíbrios musculares. Nesta amostra o limite de desequilíbrio significativo foi observado a partir de $40 \%$, com relação a incidência de patologias.

\section{NÍVEIS DE AUTO-ESTIMA E AUTO-IMAGEM EM CARDIOPATAS E SEDENTÁRIOS DE ACOR- DO COM O GÊNERO}

Kelin A. Caciatori; Neiva Leite.

Universidade Federal do Paraná-Curitiba PR.

Fundamentos: As limitações do modo de vida em indivíduos com doença cardíaca, podem estar acompanhadas de reações psicológicas que afetam a auto-estima (AE) e auto-imagem (AI). Objetivo: Avaliar os níveis de AE e AI de homens e mulheres cardiopatas em relação ao grupo controle normal. Metodologia: Estudo transversal, com 38 homens e $22 \mathrm{mu}-$ lheres, divididos em: 25 pacientes masculinos (PM), 12 pacientes femininos (PF), 13 controles masculinos (CM) e 10 controles femininos (CF). Todos responderam ao questionário de STEGLICH (1978) adaptado por STOBÄUS (1983) que avalia a AE e a AI. A comparação dos escores médios foi realizada através do teste não paramétrico de Kruskal-Wallis $(\mathrm{H})$, ao nível de significância $\mathrm{p}<0,05$. Resultados: A AI para o grupo PM foi significativamente menor do que o grupo CM $(\mathrm{p}<0,05)$. A AE não diferiu entre os mesmos. Os grupos PF e $\mathrm{CF}$ apresentaram escores muito próximos para as variáveis pesquisadas. A comparação dos escores de $\mathrm{AE}$ e $\mathrm{AI}$ entre os grupos $\mathrm{CM}$ e $\mathrm{CF}$, foram significativamente maiores nos homens em relação às mulheres $(p<0,05)$. Entre os grupos $P M$ e PF não houve diferenças significativas para AE e AI. Conclusão: Os homens cardiopatas diminuíram mais a AE e AI em relação as mulheres, a partir do controle, talvez reflexo do seu papel social. A reabilitação cardíaca deve observar os pacientes dentro de seu processo fisiológico e psicológico, abrangendo o indivíduo como um todo, melhorando a qualidade de vida pessoal e social. 


\section{O RITMO DE EXECUÇÃO AUTO-SELECIO- NADO É O MAIS APROPRIADO PARA A AVALIA- ÇÃO DA RESISTÊNCIA MUSCULAR ABDOMI- NAL}

Vitor A. Lira, Mario V. Pitaluga Filho, Kelmerson H. Buck, Marcos J. Weber, Antonio F.A. Duarte, Josué M. Moraes, André V. S. Rodrigues, Eduardo C. Martinez

Instituto da Capacitação Física do Exército (IPCFEx), RJ, Brasil.

Fundamentos: Alguns testes de resistência muscular abdominal utilizam diferentes ritmos de execução pré-estabelecidos. Objetivos: Verificar a influência de três ritmos de execução sobre o número de repetições e o rastreamento da prática regular de exercícios abdominais (PEA) e a ocorrência de incômodos lombares (OIL), com um novo procedimento de exercícios abdominais. Metodologia: Avaliou-se 54 rapazes, com idade, peso, estatura e gordura corporal de $18 \pm 0,5$ anos, $66 \pm$ $11 \mathrm{~kg}, 171 \pm 14 \mathrm{~cm}$ e $9,7 \pm 6,1 \%$ (média $\pm \mathrm{dp}$ ), respectivamente. Em três dias, três grupos aleatórios de 18 indivíduos, executaram os exercícios, de forma contrabalanceada, em ritmos auto-selecionado (R1), acelerado e lento (R2 - 60 e R3 - 20 flexões/min). A execução compreendia mãos na orelha, joelhos fletidos e as escápulas perdendo contato com o solo. Resultados: A ANOVA para medidas repetidas e o teste de Tukey demonstraram que R1 e R3 originaram mais repetições que R2 (54 \pm 24 e $52 \pm 34$ contra $42 \pm 23, \mathrm{p}<0,05)$. O teste qui-quadrado identificou diferença significativa entre $o$ esperado, em cada quartil (p25-6, p50-4, p75-4, p99-2), e a OIL, somente em R3 $(p=0,02)$. O mesmo não ocorreu entre o esperado (p25-2, p50-3, p75-4, p99-5) e a frequiência de PEA, em qualquer dos ritmos ( $p>0,11)$. Conclusão: R1 parece ser o mais indicado na avaliação da resistência muscular abdominal, já que é mais eficiente, em termos motores, e potencialmente capaz de rastrear indivíduos, considerando OIL e PEA.

\section{PRÁTICA REGULAR DE EXERCÍCIO FÍSICO: COMPARAÇÃO ENTRE OS ESTUDANTES DE MEDICINA E OUTROS UNIVERSITÁRIOS}

Yvon T. Rodrigues, Pedro P. B. Rodrigues, Eliane M. G. O. da Fonseca, Alonço C. Viana Júnior, Lúcio C.T. Araújo, Camile M. Braga, Alessandra C.G. Pedro.

Disciplina de Pediatria. Universidade Gama Filho. Faculdade de Medicina Souza Marques. RJ.

Fundamentos: A prática regular de exercícios físicos é fundamental para a manutenção da boa saúde. Apesar de possuir este conhecimento, o médico muita vezes negligencia esta prática. Objetivo: Avaliar, comparativamente, a prática de exercício físico regular pelos estudantes de medicina e de outros cursos universitários. Metodologia: Foram aplicados questionários sobre a prática de exercícios físicos a 458 alunos de medicina e 148 a alunos de outros cursos de duas universidades do Município do Rio de Janeiro. Resultados: Dentre os estudantes de Medicina, 68 (14,8\%) não responderam ao questionário, $40(8,7 \%)$ não praticam exercícios e 350
(76,4\%) exercitam-se regularmente. Dos que afirmaram praticar exercício, $99(21,6 \%)$ o fazem três vezes por semana, $106(23,1 \%)$ quatro ou mais vezes por semana.. Dentre os estudantes de outros cursos, $2(1,4 \%)$ não responderam ao questionário; 18 (12,2\%) não praticam exercícios e $128(86,5 \%)$ exercitam-se regularmente. Dos que afirmaram praticar exercício, $32(21,6 \%)$ o fazem três vezes por semana, $62(41,9 \%)$ quatro ou mais vezes por semana. Conclusão: Menos da metade dos estudantes de medicina praticam atividade física com a freqüência e regularidade necessários para o bom condicionamento físico. Entre os alunos dos outros cursos universitários este percentual é mais elevado, superando-os em quase $50 \%$.

\section{PREVALÊNCIA DE LOMBALGIA ASSOCIADA À OBESIDADE EM UMA POPULAÇÃO DE UM CENTRO UNIVERSITÁRIO}

Alexandre Peres, Adriano L. Simonato, Carolina Y. Stein, Durval R. Filho, Fernanda L. da Silva, Silvio J. C. Melhado, Sônia Barbon.

Centro Universitário de Catanduva - SP - Faculdade de Fisioterapia. Departamento de Saúde Pública e Metabolismo.

Fundamentos: A mudança do centro de gravidade em pessoas obesas tem sido relacionada a um dos fatores de dor lombar. Estudos realizados mostram a importância de se manter o valor normal do IMC (Índice de Massa Corpórea) para ajudar na preservação do estado normal e funcional da coluna. Objetivo: Avaliar a prevalência de lombalgia associada à obesidade em uma população de um Centro Universitário em Catanduva - SP. Metodologia: Foram entrevistados nessa população de 2.200 alunos; pacientes adultos, de ambos os sexos, sendo $53\left(2,4 \%\right.$ ) com IMC acima de $30 \mathrm{Kg} / \mathrm{m}^{2}$ (Obesidade tipo Grau II). Foi realizada uma entrevista semidirigida elaborada especificamente para este fim, (Inquérito Epidemiológico Obesidade x Lombalgia). Utilizou-se um gráfico-modelo padronizado de Braune e Fisher, para localização da dor (quadrantes superiores e inferiores). Através da Escala de Dor padronizada de 0 a 10 pode-se avaliar a intensidade subjetiva da mesma. Resultados: Em 53 indivíduos estudados, sendo $37(69,8 \%)$ do sexo masculino e $16(30.1 \%)$ do sexo feminino. $\mathrm{Na}$ avaliação do grupo estudado $(\mathrm{n}=53), 12(32,4 \%)$ homens e 12 (75\%) mulheres relataram, subjetivamente, lombalgia. Utilizando-se do gráfico-modelo padronizado de Braune e Fisher, os pacientes localizaram a dor nos quadrantes superiores $(58,3 \%)$ e quadrantes inferiores $(25 \%)$. Verificouse através da Escala de Dor, uma média de valor 4 nos homens e de 4,5 nas mulheres. Conclusão: Os resultados mostraram que na população universitária estudada, a prevalência de lombalgia, subjetivamente relatada, não foi associada positivamente, corroborando semelhanças estatísticas comparativas a outros trabalhos publicados. 


\section{RESPOSTA DA PERCEPÇÃO SUBJETIVA DE ESFORÇO (PSE) DE MULHERES IDOSAS SUB- METIDAS AO TESTE DE REPETIÇÕES MÁXI- MAS (RM) NO EXERCÍCIO “LEG PRESS” "45 EM DIFERENTES INTENSIDADES}

Vagner Raso, Sandra M.M. Matsudo e Victor K.R. Matsudo Centro de Estudos do Laboratório de Aptidão Física de São Caetano do Sul - CELAFISCS

Objetivo: Este estudo teve como objetivo analisar a resposta da PSE de mulheres idosas submetidas ao teste RM no exercício "leg press" $45^{\circ}$ em diferentes intensidades. Metodologia: Para tanto, a amostra foi constituída por 16 mulheres na faixa etária de 55 a 84 anos de idade (x: 65,90 \pm 7,07 anos). Os sujeitos foram semanalmente submetidos ao teste RM no exercício "leg press" $45^{\circ}$ em quatro diferentes intensidades (50\%, $60 \%, 70 \%$ e $80 \%$ ) de acordo com o teste de uma repetição máxima (1-RM). A PSE seguiu proposta de RASO et al. (2000). Foram consideradas a sobrecarga utilizada para executar o teste RM, a quantidade de repetições executadas e a PSE imediatamente após o final do teste. A ANOVA "Two Way" acompanhada do post-hoc Bonferroni e o nível de significância de p $<0,05$ foram empregados. Resultados: Evidentemente, a sobrecarga utilizada para executar o teste RM foi estatisticamente diferente independente da intensidade. Houve tendência significativa de decréscimo na quantidade de repetições executadas de acordo com o aumento da intensidade de esforço. Os valores reportados para a PSE ao final do teste RM foram similares independente da intensidade. Conclusão: Estes dados permitem concluir que: a) os sujeitos percebem o teste como sendo de característica máxima; b) existe similaridade na resposta da PSE de mulheres idosas submetidas ao teste de repetições máximas independente da intensidade; e c) os sujeitos têm maior sensibilidade para reportar escore máximo na PSE quando a intensidade de esforço é maior.

\section{AVALIAÇÃO EM MULHERES DA TERCEIRA IDADE PRATICANTES E NÃO PRATICANTES DE ATIVIDADES FÍSICAS}

Denis O. Nicoladeli; Neiva Leite.

Universidade Federal do Paraná - Curitiba (PR)

Fundamentos: No envelhecimento ocorrem diminuições das funções fisiológicas, sendo estas aceleradas pelo sedentarismo. Objetivo: Analisar os níveis de força e patologias mais freqüentes em idosas praticantes e não praticantes de atividades físicas. Metodologia: Estudo transversal, com 88 mulheres acima de 60 anos, divididas em 68 praticantes $(\mathrm{P})$ e 20 não praticantes de atividades físicas (NP). Foram coletados os hábitos de vida, doenças, pressão arterial, frequiência cardíaca, força muscular, índice de massa corporal (IMC) e relação cintura/quadril (RCQ). Resultados: O P desenvolvia atividades aeróbias, 3 vezes na semana e duração de 40 a 60 minutos. Apresentava idade média de 68,8 anos, menor que o NP com 72,30 anos $(\mathrm{p}<0,05)$. No P, $20 \%$ dos idosos não apresentavam doenças, significativamente maior que os $9,38 \%$ do NP $(\mathrm{p}<0,05)$. O P não realizava avaliações periódicas em $2,71 \%$ e o NP em $11,59 \%(\mathrm{p}<0,01)$. O P apresentou menor peso corporal $(p<0,01)$, IMC $(p<0,001)$, RCQ $(p<0,05)$, pressão arterial sistólica $(\mathrm{p}<0,05)$, aparecimento de doenças respiratórias $(\mathrm{p}<0,05)$. A força de preensão manual e de membros superiores foram discretamente maiores no $\mathrm{P}$ em relação ao NP, mas sem diferenças significativas. Conclusão: As variáveis influenciadas pelas atividades aeróbias demonstraram diferenças significativas, mas não houve melhora na força muscular, sugerindo talvez a necessidade de um trabalho muscular localizado paralelo ao condicionamento aeróbio.

\section{ANÁLISE DA DOSAGEM FARMACOLÓGICA DE PACIENTES DIABÉTICOS SUBMETIDOS À ATIVIDADE FÍSICA REGULAR}

Simone A. Dino, Adriana Rozentul, Luisa Meirelles, Vivian Liane Pinto, Pedro di Marco da Cruz.

Hospital Universitário Pedro Ernesto - Universidade do Estado do Rio de Janeiro

Objetivo: Verificar a variação na dosagem de insulina e/ou hipoglicemiantes orais administrados aos pacientes, antes e após à prática de atividade física regular. Metodologia: Foram analisados 14 pacientes diabéticos, sendo 13 do Tipo II (93\%) e 1 do Tipo I (7\%). A idade variou de 31 a 83 anos (média $=66,5$ ), onde 5 eram do sexo masculino $(35,7 \%)$ e 9 do sexo feminino (64,3\%). Resultados: Dos 50\% pacientes insulino-dependentes, $14,3 \%$ reduziram a dosagem de insulina, $57,1 \%$ mantiveram a dosagem e $28,6 \%$ aumentaram. Com relação ao hipoglicemiante oral, que correspondia a $35,7 \%$ dos pacientes, $40 \%$ reduziram a dosagem, $40 \%$ mantiveram a dosagem e $20 \%$ aumentaram. O último grupo de pacientes compreendia aqueles que faziam uso, simultâneo de insulina e hipoglicemiante oral $(14,3 \%)$. Destes $50 \%$ reduziram a dosagem, 50\% mantiveram e não houve aumento da medicação para esse grupo. Conclusão: A maioria dos pacientes manteve ou diminuiu a dosagem de medicamento. Isto demonstra provável estabilidade da doença e um possível controle do Diabetes, que representa um importante fator de risco para a doença coronariana. $\mathrm{O}$ exercício físico regular possui vários efeitos benéficos para pacientes com diabetes, por retardar e/ ou prevenir o aparecimento de patologias cardiovasculares.

\section{A GINÁSTICA LABORAL NA VISÃO DOS RE- PRESENTANTES DAS INDÚSTRIAS}

Ricardo A. Mendes, Neiva Leite, Claudia Casagrande, Herivelto Moreira.

Centro Federal de Educação Tecnológica, Curitiba, PR

Fundamentos: A ginástica laboral (GL) apareceu nas empresas modernas como prevenção, reabilitação de doenças relacionadas com o trabalho e para melhorar a qualidade de vida dos trabalhadores. Objetivos: Determinar as indústrias da Cidade Industrial de Curitiba (CIC) que aplicavam o progra- 
ma de GL em seus trabalhadores e verificar os benefícios que a GL traz às indústrias e aos trabalhadores na visão dos representantes das indústrias. Metodologia: Estudo transversal, quantitativo e descritivo dividido em 2 fases. $\mathrm{Na} 1^{\mathrm{a}}$ fase, utilizou-se a "Relação das empresas instaladas na CIC por setores" e na $2^{\mathrm{a}}$ foi confeccionado um questionário (questões objetivas e fechadas), levado em mãos e aplicado pelos pesquisadores nos representantes das indústrias que possuíam a GL. Resultados: A GL era ministrada em $8(3,11 \%)$ das 257 indústrias da CIC. Quanto aos benefícios para os trabalhadores: houve uma melhoria na qualidade de vida (33\%), melhora de bem-estar geral (17\%), aumento da saúde (17\%), diminuição da fadiga muscular (17\%) e aumento no desempenho do trabalho (16\%). Quanto aos benefícios encontrados nas indústrias: houve uma diminuição das doenças ocupacionais dos trabalhadores (33\%) e diminuição dos acidentes de trabalho (50\%). Conclusão: Parece existir pouco interesse das indústrias da CIC em investir financeiramente na implantação da GL, talvez porque os resultados e os benefícios da GL sejam mais relevantes e significativos para os trabalhadores (melhoria da saúde) e somente indiretos e a longo prazo para as indústrias.

\section{ANÁLISE QUALITATIVA DOS ESPESSÍME- TROS DE DOBRAS CUTÂNEAS}

Christiane L Corrêa, Astrogildo V. de Oliveira Jr.

Laboratório de Cineantropometria da Universidade do Estado do Rio de Janeiro, IEFD, Universidade do Estado do Rio de Janeiro, Rio de Janeiro.

Fundamentos: Um dos métodos mais difundidos para a predição da composição corporal, é o método de dobras cutâneas. Por sua fácil utilização e interpretação vem sendo largamente empregado. No entanto, pesquisas demonstraram diferenças nas medidas obtidas com diferentes tipos de adipômetros, as quais podem afetar significantemente o resultado final, prejudicando a fidedignidade da análise desses dados. Uma das causas apontadas para estas diferenças relaciona-se a falta de manutenção. Um aparelho pode ser usado durante anos sem que, ao menos, uma verificação na sua calibragem seja feita. Objetivo: Determinar as condições de uso de adipômetros utilizados em academias no município do Rio de Janeiro. Metodologia: Foram analisados 30 adipômetros, (14 cescorf científicos; 10 lange; 03 sanny; 01 harpenden; 01 body caliper; 01 cescorf plástico). Observou-se o tempo de uso, a frequiência de utilização, danos aparentes e considerações acerca do aspecto físico dos mesmos. Resultados: Cinco adipômetros apresentaram alguma imprecisão para suas utilizações, três apresentavam irregularidades nas pressões das molas e um apresentava-se de manuseio difícil, limitando a profundidade de abordagem da dobra cutânea. A média de tempo de utilização foi 2,6 anos $( \pm 1,1)$ e a freqüência semanal média de uso de 10,7 $( \pm 5,8)$ avaliações por semana, sendo que $100 \%$ dos instrumentos jamais passaram por uma calibragem ou qualquer outro serviço de manutenção. Conclusão: A quali-

Rev Bras Med Esporte - Vol. 7, № 3 - Mai/ J un, 2001 dade para uso dos instrumentos estudados encontra-se comprometida e que urge que se criem formas e serviços especializados para a manutenção destes aparelhos.

\section{DESENVOLVIMENTO MATURACIONAL EM ATLETAS DE NATAÇÃO INFANTO JUVENIS}

Eva L. da Silva, Gilmara Chiamulera, Neiva Leite, Ricardo A. Mendes, Paulo César B. Bento.

Centro Universitário Positivo - UnicenP - Curitiba, PR

Fundamentos: A variação da maturidade biológica influencia no desempenho físico de forma positiva ou negativa, de acordo com a modalidade esportiva realizada. Objetivo: Avaliar o grau de maturação em nadadores de 12 a 16 anos, participantes do Sul Brasileiro de Natação no ano 2000. Metodologia: Estudo transversal que avaliou 257 atletas, 118 femininos (fem) e 139 masculinos (masc). Todos se auto-avaliaram conforme estágios de desenvolvimento de Tanner, e a cada 05 atletas foi realizado um exame confirmatório. Resultados:

\begin{tabular}{lc|c|c|c|c|c|c}
\hline \hline Estagio & Idade & 12 & 13 & 14 & 15 & 16 & $\begin{array}{c}\text { total } \\
n\end{array}$ \\
\cline { 1 - 7 } Fem. & $n$ & 19 & 40 & 20 & 30 & 09 & \\
& $\mathrm{M} 4+\mathrm{M} 5$ & $17 \%$ & $57 \%$ & $55 \%$ & $79 \%$ & $08 \%$ & 118 \\
& $\mathrm{P} 4+\mathrm{P} 5$ & $39 \%$ & $65 \%$ & $86 \%$ & $93 \%$ & $09 \%$ & \\
\hline \multirow{2}{*}{ Masc. } & $n$ & 13 & 33 & 37 & 42 & 15 & \\
& $\mathrm{G} 4+\mathrm{G} 5$ & $31 \%$ & $52 \%$ & $58 \%$ & $77 \%$ & $93 \%$ & 139 \\
& $\mathrm{P} 4+\mathrm{P} 5$ & $58 \%$ & $53 \%$ & $89 \%$ & $93 \%$ & $100 \%$ & \\
\hline
\end{tabular}

Conclusão: A prescrição do treinamento deve respeitar o grau maturacional. Os nadadores ao atingirem os estágios 4 e 5 serão beneficiados com o aumento da força principalmente nos meninos e melhora da flutuabilidade nas meninas.

\section{MÉTODO O-SCALE NA AVALIAÇÃO DO PER- FIL CORPORAL DE PACIENTES COM HIPERTI- REOIDISMO}

Kelb B. Santos, Rubens A.C. Filho, Ney D. Barreto, Mario Vaisman, Bruno A. Salvador, Andréia M.O. Souza, Antonio C. L. Nóbrega

Universidade Federal Fluminense e Univ. Fed. do Rio de Janeiro, RJ.

Fundamentos: $O$ hipertireoidismo cursa com fraqueza e atrofia muscular esquelética generalizada. O método $O$-Scale é um método de avaliação do perfil corporal que apresenta uma descrição geral através do índice A (adiposidade) e W (peso proporcional) cuja diferença estima o desenvolvimento músculo-esquelético. Objetivo: Descrever o perfil corporal de pacientes com hipertireoidismo através do método $O$-Scale. Metodologia: Doze pacientes ( $8 \mathrm{fem}, 4$ masc; idade $43 \pm 3$ anos) com tireotoxicose clínica e laboratorial foram submetidos à antropometria incluindo: estatura, massa corporal, circunferências (braço relaxado e fletido tensionado, antebraço, punho, tórax, cintura, glúteo, coxa, perna e tornozelo), dobras cutâneas (biciptal, triciptal, subescapular, suprailíaca, supraespinhal, abdmominal, coxa e perna medial) e diâmetros ósseos (umeral e femural). Os dados foram inseridos em um programa de computador (O-Scale System, Rosscraft, Cana- 
da) que gera os índices $\mathrm{A}$ e $\mathrm{W}$ a partir do reescalonamento do somatório de seis dobras cutâneas e da massa corporal para uma altura padrão de $170,18 \mathrm{~cm}$ e os classifica em uma escala de 9 categorias utilizando um banco de dados de indivíduos do mesmo sexo e idade. Resultados: Índice A [mediana (diferença interquartil) $]=7,5$ (5); índice $\mathrm{W}=6$ (3). Nove pacientes $(75 \%)$ apresentaram $\mathrm{W}$ menor que A denotando um hipodesenvolvimento muscular esquelético. Conclusão: $\mathrm{O}$ método $O$-Scale foi capaz de demonstrar o comprometimento muscular esquelético típico do hipertireoidismo.

\section{EFEITOS DA SUPLEMENTAÇÃO DE CREATI- NA MONOHIDRATADA NA COMPOSIÇÃO COR- PORAL DE MULHERES NÃO SEDENTÁRIAS}

Silvana R. U. Lobato, Ana Beatriz M. de C. Monteiro, Luiz B. Mercanti, Rogério M. Duarte, José F. Filho.

Universidade Castelo Branco, RJ/RJ.

Fundamentos: A creatina monohidratada é um dos mais recentes suplementos nutricionais (PEETERS \& cols., 1999). Sua suplementação pode promover mudanças na composição corporal (KREIDER, 1998). Objetivo: Verificar os efeitos da suplementação de creatina monohidratada na composição corporal de mulheres não sedentárias. Metodologia: 10 mulheres entre 20 e 35 anos não sedentárias, submetidas ao treinamento contra resistência durante 60 dias, sendo que 5 ingeriram creatina e 5 não ingeriram. Foram medidos peso total, estatura, 7 dobras cutâneas, IMC, peso magro e peso total de água. Para o cálculo do percentual de gordura o protocolo utilizado foi POLLOCK 7 dobras e para o peso total de água o aparelho FUTREX 5000. Entende-se que p $<0,05$, para H0: não há diferença estatística significativa entre os testes, $?_{1}=$ $? ?$ ? para H1: há diferença estatística significativa entre os testes,?, < >?. Resultados: No grupo com creatina aceitou-se H1 para peso total, peso magro, IMC e peso total de água e rejeitou-se H0. Para o grupo sem creatina aceitou-se H0 e rejeitou-se H1. Conclusões: Pôde-se concluir que os indivíduos que fizeram uso da creatina, apresentaram uma evolução maior que os demais, particularmente no condizente à peso total, IMC, peso magro e peso total de água.

\section{MÉTODOS PARA A PREDIÇÃO DO PERCEN- TUAL DE GORDURA (\% GC): ESTUDO COMPA- RATIVO EM FUZILEIROS NAVAIS DO BATA- LHÃO TONELEIRO DA CIDADE DO RIO DE JA- NEIRO (BRASIL)}

Mônica S. Torres, Maria S.C. Sousa, Miguel de Arruda, Idico L. Pellegrinotti

Centro Universitário da Cidade; UFPB; Unicamp

Fundamentos: O percentual de gordura corporal $(\% \mathrm{GC}) \mathrm{de}-$ corre de um parâmetro que atualmente tem sido estudado em diversas regiões e diferentes populações. Objetivo: Verificar qualitativamente e quantitativamente a existência ou não de diferenças entre os protocolos para cálculo\%GC, segundo as equações de Faulkner (Rizzo, 1977) (F), Jackson \& Pollock (1978) (J\&P) e Guedes (1989) (G), e o protocolo de Dotson $\&$ Davis (Torres, 1998) (D\&D). Metodologia: O estudo transversal, analítico, amostra não-probabilística, dados primários mensurou 47 fuzileiros navais do Batalhão Toneleiro da Cidade do Rio de Janeiro, média de idade 25,6 $( \pm 4,7)$ anos, estatura $174,6( \pm 5,2) \mathrm{cm}$, peso $74,4( \pm 7,04)$. Os dados constituíram a matriz analítica das médias, desvio-padrão e variâncias. Acatamos o nível de significância de 0,05. Selecionamos o Teste "t" de Student e a correlação "r" de Pearson. Resultados: Não há diferenças significativas $(\mathrm{p}=0,8349)$ entre os resultados para o\%GC, nos seus valores absolutos, calculados pelos protocolos $\mathbf{F}(13,8 \% \pm 3,23)$ e $\mathbf{D} \& \mathbf{D}(13,9 \% \pm 3,60)$; há diferenças significativas $(\mathrm{p}=0,000-17)$, entre os protocolos de $\mathbf{J} \& \mathbf{P}(8,6 \% \pm 4,50)$ e $\mathbf{D} \& \mathbf{D}$, há diferenças significativas $(\mathrm{p}=0,0005,77)$ entre os protocolos $\mathbf{G}(16,9 \% \pm 5,85)$ e $\mathbf{D} \& \mathbf{D}$. Na correlação utilizada, o estudo observou o cálculo de normatização, em que todos os protocolos apresentaram coeficiente de determinação superior a $0,50(50 \%)$ e positivos, $\mathbf{F}$ e $\mathbf{D \& D}$ r = 0,735; J\&P e D\&D r = 0,770; G e D\&D r = 0,734. Conclusões: Os protocolos apresentam uma diferença de fase, derivada das particularidades de cada um; discordam quanto ao valor absoluto da medida, contudo são paralelos. Recomendamos o uso de medidas de circunferências para o cálculo do\% GC em homens.

\section{RELAÇÃO ENTRE PERCENTUAL DE GOR- DURA E ATIVIDADE FÍSICA RELATADA EM FREQÜENTADORES DA PRAIA DE COPACABA- NA - RIO DE JANEIRO}

Vanessa Périssé, Hebe F. Cordeiro, Tony M. dos Santos Laboratório de Fisiologia do Exercício - Universidade Estácio de Sá

Fundamentos: Evidências epidemiológicas sugerem uma associação inversa entre quantidade de atividade física praticada e percentual de gordura corporal (USDHHS, 1996). Objetivo: Estabelecer a influência da prática de atividade física sobre a magnitude da gordura corporal numa amostra da população do RJ. Metodologia: mil quatrocentos e quarenta e nove voluntários, na faixa etária de 18 a 67 anos, sendo $35 \%$ do sexo masculino e $65 \%$ do sexo feminino, forneceram informações acerca de seus hábitos de atividade física $(C D C$, 1997), além de serem submetidos a uma avaliação para determinação da composição corporal (método de Pollock). Resultados: Nos homens, o percentual de gordura corporal observado foi significativamente $(\mathrm{p}<0.05)$ mais elevado nos indivíduos inativos $(20.8 \pm 7.94 \%)$ ou praticantes de atividades leves/moderadas $(21.0 \pm 7.39 \%)$ em relação aos praticantes de atividades vigorosas $(16.5 \pm 6.33 \%)$ e contra-resistência $(16,8 \pm 6.6 \%)$. Na população feminina, foram encontrados níveis mais elevados de gordura corporal em indivíduos inativos $(25.0 \pm 6.9 \%)$ ou praticantes de atividades leves/moderadas $(24.7 \pm 6.4 \%)$ do que em atividades vigorosas $(22.7 \pm$ $7.5 \%$ ) e nas atividades contra-resistência, porém sem signifi- 
cância estatística $(\mathrm{p}<0.05)$. Conclusão: Os resultados sugerem que em atividades vigorosas existe uma melhora no controle da gordura corporal. Considerando os riscos associados a essas atividades, sua prática pode proporcionar benefícios no controle do peso corporal.

\section{AVALIAÇÃO ERGOESPIROMÉTRICA DOS EFEITOS DO TREINAMENTO AERÓBIO EM GESTANTES COM SOBREPESO}

Ricardo Stein, Iracema A. Santos, Sandra C. Fuchs, Bruce B. Duncan, Maria I Schmidt, Luciana Nucci, Cristiane Borba, Jorge P. Ribeiro

Serv.Cardiologia HCPA-UFRGS,Depto. Med.Social-FAMED-POA-RS

Introdução: $\mathrm{O}$ exercício tem sido indicado para mulheres grávidas, porém, alterações fisiológicas gestacionais tornam necessárias adaptações, tanto nos protocolos de avaliação quanto nos programas de atividade física a serem prescritos.O teste ergoespirométrico(TE) permite mensurar a capacidade de trabalho físico prolongado em gestantes. Objetivo:Avaliar os efeitos do treinamento aeróbio sobre a capacidade funcional em gestantes de baixo risco que apresentam sobrepeso através de TE submáximo ( $\mathrm{TE}_{\mathrm{Smax}}$ ) em esteira. Métodos:Através de ensaio clínico randomizado estudou-se 22 gestantes hígidas com sobrepeso, sedentárias com idade gestacional [ 20 semanas e idade $\mathrm{m}$ a 20 anos. Onze gestantes foram randomizadas para o grupo intervenção (GI), realizando exercícios aeróbios 3 vezes por semana, 40 minutos por sessão, em uma FC medida por freqüencímetro de $140 \mathrm{bpm}$. As onze gestantes do grupo controle (GC) receberam cuidados pré-natais convencionais. Através de protocolo de rampa uniforme, $\mathrm{TE}_{\mathrm{S} \text { - }}$ ${ }_{\max }$ foi utilizado na entrada, sendo repetido após 12 semanas. De forma cega, o $\dot{V O}_{2 \text { limiar }}$ foi identificado em cada $\mathrm{TE}_{\text {Smax }}$ por 2 cardiologistas proficientes no método. Anova de dupla entrada para medidas repetidas foi utilizada. Resultados:Em ambos os grupos, o peso aumentou de forma semelhante ao longo do experimento. Houve aumento de $18 \%$ no $\dot{\mathrm{VO}}_{2 \text { limiar }}(\mathrm{P}<0,05)$ entre o primeiro e o segundo $\mathrm{TE}_{\text {Smax }}$ no GI e nenhuma diferença no GC.

\begin{tabular}{l|c|c|c}
\hline & $\left.\mathrm{VO}_{2 \text { limiar teste I (ml.kg.min) }}\right)^{1}$ & $\mathrm{VO}_{\left.2 \text { limiar teste II (ml.kg.min })^{1}\right)}$ & $\mathrm{p}$ \\
\hline GC & $16 \pm 3$ & $16 \pm 3$ & $\mathrm{NS}$ \\
\hline GI & $17 \pm 3$ & $20 \pm 3$ & $<0,05$ \\
\hline
\end{tabular}

Conclusões: A despeito do aumento na idade gestacional e no peso corporal, as gestante expostas a um programa de treinamento aeróbio de 12 semanas apresentaram um incremento significativo na capacidade funcional avaliada pela medida do limiar anaeróbio determinado por TE. (Apoio: CAPES, PRONEX, CNPq, Bristol-Myers Squibb Foundation).

\section{EFEITO DA DIETA HIPOCALÓRICA E DO TREINAMENTO FÍSICO NA CAPACIDADE FUN- CIONAL EM MULHERES OBESAS}

Isabel C.D. Ribeiro, Ivani C. Trombetta, Luciana Batalha, Maria U.P.B. Rondon, Fátima H.S. Kuniyoshi, Antonio C. P. Barretto, Sandra Villares, Carlos E. Negrão.
Instituto do Coração (InCor) HC-FMUSP, São Paulo, SP.

Fundamentos: Redução de peso corporal pode melhorar a capacidade funcional e diminuir a pressão arterial (PA) de repouso. Entretanto, a magnitude dessas alterações decorrentes da dieta hipocalórica comparada com a dieta hipocalórica associada ao treinamento físico ainda precisa ser melhor documentada. Objetivo: Comparar os efeitos da dieta hipocalórica com os efeitos da dieta hipocalórica associada ao treinamento físico sobre a PA de repouso e capacidade aeróbia de pico em mulheres obesas. Metodologia: Foram estudadas 23 mulheres obesas, normotensas subdivididas em 2 grupos: 1 . Dieta hipocalórica por 16 semanas (D, 33 2 anos, IMC $34 \pm 2 \mathrm{~kg} / \mathrm{m}^{2}, \mathrm{n}=10$ ) e 2 . Dieta mais treinamento físico por 16 semanas (DTF $34 \pm 7$ anos, IMC $33 \pm 2 \mathrm{~kg} / \mathrm{m}^{2}, \mathrm{n}=13$ ). A capacidade funcional foi avaliada através de protocolo de rampa, com incremento de 10-15W. A PA foi avaliada pelo método auscultatório. Resultados: Não houve diferença significativa na perda de peso corporal entre os grupos (DTF $=-6,4 \pm 0,5$ vs $\mathrm{D}=-7,2 \pm 0,5 \mathrm{~kg}, P<0,6) . * P<0,05$ ( 1 entre grupos)

\begin{tabular}{lccccccc}
\hline & $\mathrm{FC}$ & $\mathrm{PAS}$ & $\mathrm{PAD}$ & \multicolumn{2}{c}{$\mathrm{VO}_{2}$ repouso } & \multicolumn{2}{c}{$\mathrm{VO}_{2}$ pico } \\
& $(\Delta \%)$ & $(\Delta \%)$ & $(\Delta \%)$ & $\mathrm{ml} / \mathrm{kg} / \mathrm{mi}$ & $(\Delta \%)$ & $\mathrm{ml} / \mathrm{kg} / \mathrm{mi}$ & $(\Delta \%)$ \\
& & & & $\mathrm{n}$ & & $\mathrm{n}$ & \\
\hline DTF & $-1,8 \pm 4,7$ & $1,0 \pm 3,6$ & $2,2 \pm 3,7$ & $3,5 \pm 0,1$ & $13,1 \pm$ & $23,0 \pm$ & $14,6 \pm$ \\
& & & & & 10,0 & $1,0^{*}$ & $3,9^{*}$ \\
$\mathrm{D}$ & $-7,7 \pm 4,8$ & $0,4 \pm 2,5$ & $-6,6 \pm 3,5$ & $3,4 \pm 0,2$ & $-1,6 \pm 8,8$ & $18,7 \pm$ & $1,5 \pm 4,3$ \\
& & & & & & 1,3 & \\
\hline
\end{tabular}

Conclusão: A D e DTF provocam perda de peso semelhante em mulheres obesas normotensas, mas não modificam a PA de repouso. A DTF, ao contrário da $\mathrm{D}$, provoca aumento expressivo na capacidade aeróbia máxima em mulheres obesas.

42 EFEITOS DO TREINAMENTO CONTRA RESISTÊNCIA SOBRE A FORÇA MUSCULAR E O DESEMPENHO DE HABILIDADES FUNCIONAIS SELECIONADAS EM MULHERES IDOSAS

Amandio A. R. Geraldes, Jani C. B. de Aragão. Estélio H. M. Dantas.

PROCIMH, Universidade Castelo Branco, Rio de Janeiro, RJ.

Fundamentos: $\mathrm{O}$ declínio da força e da massa muscular durante o envelhecimento tem sido relacionado à fragilidade física, declínio funcional, mobilidade deficiente e quedas. Objetivos: Verificar os efeitos do treinamento contra resistência (TCR) sobre a força muscular e o desempenho das habilidades funcionais selecionadas (HFS) em mulheres idosas. Metodologia: Estudo do tipo experimental com amostra composta por 28 mulheres com idades entre 60 e 74 anos, acompanhadas por oito semanas em três sessões semanais, de 60 minutos, em dias alternados. Para avaliação da força dinâmica máxima, utilizou-se o teste de uma repetição máxima (1RM); para a avaliação do desempenho HFS, utilizou-se o tempo mínimo para a realização das seguintes tarefas: a) caminhar 10 metros; b) levantar-se da posição decúbito dorsal; c) "Timed Up \& Go Test"; d) levantar-se de uma cadeira cinco vezes. Resultados: Adotou-se um nível de significância 
de $\mathrm{p}<0,05$ em todos os casos. No grupo experimental, observaram-se melhoras significativas na força muscular e no desempenho na HFS (com exceção do "Timed Up \& Go Test"; $p$ $=0,0939$ ). Igualmente, houve correlações significativas entre diversos parâmetros de força muscular e as HFS ( $\mathrm{r} \geq 0,30$; $\mathrm{p}<0,05)$. Conclusão: Os resultados indicam que, além de seguros, os programas de TCR quando aplicados à população de idosas, possibilitam melhoras significativas na força muscular e no desempenho das HFS, o que pode contribuir com maiores autonomia e independência funcional.

\section{ERGOESPIROMETRIA NA INSUFICIÊNCIA CARDÍACA CRÔNICA: QUALIDADE DE VIDA E CONSUMO DE OXIGENNIO CORRIGIDO PELA MASSA MUSCULAR}

Ricardo Vivacqua, Salvador M.Serra, Antonio Cláudio L.Nóbrega, Salete J.F.Rego, Armando Cantisano, Maurício Wajngarten. Instituições: Incor FMUSP SP, UFF RJ, Hospital Procardíaco RJ.

Fundamento: Em portadores de insuficiência cardíaca (IC) os índices de qualidade de vida (IQV) se relacionam à capacidade funcional. Objetivo: verificar a influência da massa muscular esquelética (MME) na relação entre a capac.funcional e o IQV em pac. com IC. Mtodologia: 11 homens sedent. com IC de etiol. não isquêmica, classe func.III NYHA, $47 \pm 12$ a. índice de massa corporal: $24,3 \pm 2,9 \mathrm{~kg} / \mathrm{m}^{2}$, fr. de ejeção (eco): $25 \pm 7 \%$, MME de panturrilhas e coxas (ressonância): $5,7 \pm 0,7 \mathrm{~kg}$, foram submetidos ao questionario de Minessota adaptado e a 2 testes ergoespirométricos em esteira rolante, protocolo de rampa, limitados por fadiga ou dispnéa, sendo o $1^{\circ}$ teste para adaptação e determinação da capac. funcional. Os valores do consumo de oxigênio $\left(\mathrm{V}_{2}\right)$ no limiar anaeróbio (LA) e no pico do esforço $\left(\mathrm{V}_{2}\right.$ pico) foram corrigidos pela massa corporal total (/MC) e pela MME de membros. Inferiores. Resultados: $\mathrm{V}_{2}$ pico/MC: $18,3 \pm 6,6 \mathrm{ml} /$ $\mathrm{kg} / \mathrm{min} ; \dot{\mathrm{V}} \mathrm{O}_{2} \mathrm{LA} / \mathrm{MC}: 11,9 \pm 3,1 \mathrm{ml} / \mathrm{kg} / \mathrm{min} ; \dot{\mathrm{VO}}_{2}$ pico / MME: $22,3 \pm 9,9 \mathrm{ml} / \mathrm{kg} / \mathrm{min} ; \mathrm{V}_{2}$ LA $/ \mathrm{MME}: 15,5 \pm 5,1 \mathrm{ml} / \mathrm{kg} /$ $\mathrm{min}$; IQV escore:51 \pm 24 . Correlações lineares entre as variáveis: $\mathrm{IQV} \times \dot{\mathrm{VO}}_{2}$ pico /MC: $\mathrm{r}=-0,47(\mathrm{P}>0,05)$; IQV x $\dot{\mathrm{VO}}_{2}$ pico /MME: $\mathrm{r}=-0,62(\mathbf{P}<\mathbf{0 , 0 5})$; IQV x VंO ${ }_{2}$ LA /MC: $\mathrm{r}=-$ 0,37 (P>0,05); IQV x $\dot{V}_{2}$ LA /MME: $\mathrm{r}=-0,22(\mathrm{P}>0,05)$. Conclusão: nos portadores de IC a MME influi e deve ser considerada na avaliação da relação entre capac.funcional e IQV.

\section{EXERCÍCIO E VARIABILIDADE DA FRE- QÜÊNCIA CARDÍACA EM INDIVÍDUOS NOR- MAIS E CHAGÁSICOS: ESTUDO PILOTO}

Pedro P. Pereira Jr., L. A. Imbiriba, F. C. Jandre, Fátima P. Oliveira. Escola de Educação Física e Desportos - UFRJ, RJ

Fundamentos: $\mathrm{O}$ espectro de potência da série de intervalos entre as ondas $\mathrm{R}$ do eletrocardiograma (ECG), indica a influência e a interação dos componentes simpático e parassimpático do sistema nervoso au-tônomo sobre a regulação da frequiência cardíaca. Objetivo: Analisar a Variabilidade da Freqüência Cardíaca (VFC) em indivíduos normais e em cardiopatas chagásicos crônicos, durante 6 situações: repouso$T 1$, aquecimento-T2, exercício (ponto médio-T3 e pico-T4), e recuperação pós-exercício (minuto 2-T5 e minuto 4-T6). Metodologia: 10 voluntários do sexo masculino, sendo 5 normais (34,8 $\pm 9,42$ anos) e 5 chagásicos ( $42 \pm 7,25$ anos) foram submetidos a um teste máximo em cicloergômetro (WHIPP et al., 1981). Os sinais de ECG (derivação DII) foram coletados com frequiência de amostragem de $240 \mathrm{~Hz}$, utilizando um conversor $\mathrm{A} / \mathrm{D}$ de 12 bits. $\mathrm{O}$ cálculo da razão média entre baixa frequiência e alta freqüência (BF/AF) da série de intervalos RR foi realizado em cada trecho analisado (duração: $60 \mathrm{~s}$ ). Resultados: Os resultados medianos para cada estágio e para os dois grupos avaliados estão apresentados na tabela abaixo:

$\begin{array}{lllllll}\text { Grupos } & \mathrm{T} 1 & \mathrm{~T} 2 & \underline{\mathrm{T}} & \mathrm{T} 4 & \mathrm{~T} 5 & \mathrm{~T} 6 \\ \text { Normais } & 0,43 & 0,72 & 1,44 & 0,78 & 0,40 & 0,68 \\ \text { Chagásicos } & 0,30 & 0,39 & 0,50 & 0,30 & 0,13 & 0,39\end{array}$

Conclusão: A técnica utilizada indicou diferenças qualitativas na regulação da freqüência cardíaca entre os grupos e nos estágios ana-lisados. Entretanto, diferenças estatísticas neste estágio inicial do estudo não foram testadas devido à pequena amostra utilizada, porém o estudo da VFC parece discriminar alterações autonômicas. CNPq e FAPERJ.

\section{INFLUÊNCIA DO TREINAMENTO FÍSICO AERÓBIO NA RESPOSTA AO TESTE ERGOMÉ- TRICO DE PACIENTES DO NÚCLEO DE PRE- VENÇÃO E REABILITAÇÃO CARDÍACA}

Luísa R. de Meirelles; Adriana Rozentul; Simone Dino; Vivian L.M. Pinto; Pedro di Marco da Cruz.

Hospital Universitário Pedro Ernesto - UERJ - Brasil

Objetivo: Analisar a resposta de variáveis fisiológicas durante Teste Ergométrico(TE), em resposta à prática regular e supervisionada de exercícios aeróbios após 3, 6 e 12 meses. Metodologia: Foram selecionados 199 pacientes, sendo 61 coronariopatas crônicos, 63 hipertensos, 27 coronariopatas e hipertensos, 23 diabéticos e 25 dislipidêmicos, dos quais 101 eram homens $(51 \%)$, com média de idade de 57,1 anos, que realizaram atividade física aeróbia regular. Analisou-se o comportamento da frequêencia cardíaca $(\mathrm{FC})$ de repouso e máxima, pressão arterial sistólica (PAS) e diastólica (PAD) de repouso e máxima, tempo de esforço, consumo máximo de oxigênio $\left(\mathrm{V}_{2} \mathrm{SL}\right.$ ), equivalente metabólico (MET) e duplo produto (DP), dados estes obtidos a partir de quatro TE realizado em tapete rolante segundo o Protocolo de Bruce. Resultados: A avaliação dos resultados demonstrou os benefícios do exercício, caracterizados pelo aumento do tempo de esforço, do $\dot{\mathrm{V}}_{2} \mathrm{SL}$ e do MET $(\mathrm{p}<0,01)$. Houve redução da FC de repouso, decréscimo da PAS de repouso nos hipertensos já nos três primeiros meses de treinamento. Conclusão: A diferença observada confirma os valores encontrados na literatura, colocando a prática regular de exercícios físicos como parte inte- 
grante dos meios terapêuticos disponíveis na prevenção primária e secundária da doença arterial coronariana.

46 A FORÇA MUSCULAR DINÂMICA ISOTÔNICA E PICOS DE TORQUE ISOCINÉTICO E ISOMÉTRICO DURANTE O PERÍODO DE DESTREINAMENTO DE FORÇA EM MENINOS PRÉ-PÚBERES

Andrea S. da Fontoura e Flávia Meyer.

Escola de Educação Física - Universidade Federal do Rio Grande do Sul, Laboratório de Pesquisa do Exercício - LAPEX - Porto Alegre, RS.

Fundamentos: A treinabilidade de força em crianças tem sido bastante explorada, mas ainda existe um questionamento: $\mathrm{O}$ quanto a força decresce quando a criança interrompe o treinamento? Objetivo: avaliar a magnitude da perda de força muscular dinâmica isotônica (1-RM), picos de torque isocinético e isométrico, na extensão de joelho (EJ) e flexão de cotovelo (FC) de meninos durante 12 semanas de destreinamento. Metodologia: Um grupo experimental (EX) de 7 meninos $(9,4 \pm 1,6$ anos) pré-púberes treinou de forma dinâmica, três vezes por semana, durante 12 semanas com intensidade entre 60 e $85 \%$ do teste de 1-RM, obtendo um aumento de $78 \%$ e $67 \%$ na força de 1-RM da EJ e da FC respectivamente. No grupo controle $(\mathrm{CO})$ participaram 7 meninos pré-púberes pa-

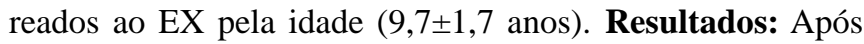
12 semanas de destreinamento, a força absoluta de 1-RM da EJ e da FC do grupo EX apresentou uma queda estatisticamente não significativa de $33 \%$ e $21 \%$, respectivamente. Quando corrigida pelo peso corporal e massa corporal magra (MCM), a força do grupo EX de 1-RM da EJ diminuiu $41 \%$ e $36 \%(\mathrm{p}<0,05)$ respectivamente. Na FC a força não apresentou redução significativa. Independente do grupo, os picos de torque não apresentaram alterações significativas com o treinamento e destreinamento. $\mathrm{O}$ grupo $\mathrm{CO}$ não alterou significativamente a força nas primeiras 12 semanas, mas ao final das 24 , aumentou a força de 1-RM em $41 \%$ e $53 \%$ na EJ e FC, respectivamente. Conclusão: No destreinamento, a queda de força de 1-RM foi significativa em valores corrigidos pelo peso corporal e MCM, apenas nos membros inferiores. O processo de crescimento e maturação pode contribuir para tornar menos evidente a redução da força durante o destreinamento.

47 ANÁLISE DO DESENVOLVIMENTO DA FORÇA MUSCULAR DE MEMBROS INFERIORES EM JOGADORES DE FUTEBOL AMADOR DA $1^{\text {a }}$ DIVISÃO DO ESTADO DO RIO DE JANEIRO, ENTRE AS CATEGORIAS MIRIM, INFANTIL, JUVENIL E JÚNIOR

Paulo N. Silva, Nayra Halm, Paulo H. N. Costa, Marceli O. Nascimento.

Depto. de Futebol Amador, Clube de Regatas do Flamengo, RJ.

Fundamentos: As mudanças fisiológicas e estruturais do impulso de crescimento na adolescência refletem-se no incre-

Rev Bras Med Esporte - Vol. 7, № 3 - Mai/J un, 2001 mento da força muscular e da massa livre de gordura ou LBM. A relação entre essas variáveis é um fator importante no controle do desenvolvimento durante a adolescência. Objetivo: Comparar a evolução da força muscular de membros inferiores (m.i.) por Kg de LBM entre as diferentes categorias desta amostra. Metodologia: A amostra constituiu-se de 71 atletas do sexo masculino, jogadores de futebol amador da $1^{\text {a }}$ divisão do estado do Rio de Janeiro. A mensuração da força dos membros inferiores foi obtida em um aparelho isocinético da marca Cybex, modelo Norm. A avaliação foi precedida de aquecimento em cicloergômetro por 8 minutos. Os atletas realizaram 3 repetições a $60 \%$ s para reconhecimento do aparelho e em seguida foram submetidos ao teste propriamente dito, que consistiu em 5 repetições de extensão e flexão de joelho com força máxima e na velocidade angular de $60 \%$ s. Resultados: Os resultados apresentados na tabela, correspondem aos valores médios de m.i.d. e m.i.e., por categoria:

\begin{tabular}{|c|c|c|c|c|}
\hline Categoria & $\underline{N}$ & $\frac{\mathrm{Nm} / \mathrm{Kg} \mathrm{de}}{\mathrm{LBM}}$ & $\frac{\text { Desvio }}{\text { Padrão }}$ & $\begin{array}{l}\text { Ganho de Nm/ Kg } \\
\text { de LBM }\end{array}$ \\
\hline Mirim & 5 & 2,932 & 0,300 & - \\
\hline Infantil & 22 & 3,576 & 0,594 & 0,644 \\
\hline Juvenil & 22 & 4,048 & 0,419 & 0,472 \\
\hline Júnior & 22 & 4,175 & 0,402 & 0,127 \\
\hline
\end{tabular}

Conclusão: Na amostra estudada, verificou-se ganhos de força entre todas as categorias. Os resultados obtidos estão em consonância com os encontrados na literatura específica; os quais ratificam o desenvolvimento da força com o aumento da idade.

48 ANÁLISE DA RELAÇÃO DO TESTE DE IMPULSÃO VERTICAL COM A FORÇA MÁXIMA DE EXTENSÃO DOS JOELHOS EM APARELHO ISOCINÉTICO EM JOGADORES DE FUTEBOL AMADOR DA PRIMEIRA DIVISÃO DO RIO DE JANEIRO

Bruno B. Costa, João Pedro Castro, Roberto M. Cascon e Ana L. Komora

Fundamentos: A avaliação da força muscular em membros inferiores é fundamental na preparação de jogadores de futebol. O método isocinético fornece dados fidedignos a respeito. Porém, o alto custo destes equipamentos o inviabiliza para a maioria dos clubes brasileiros. Objetivos: Comparar a força máxima de extensão de joelhos com o teste de impulsão vertical em jogadores de futebol amador. Procurar alternativas de testes de campo para estimar valores de força obtidos em aparelho isocinético. Metodologia: 44 atletas entre 14 e 17 anos foram submetidos a um teste de força muscular em aparelho Cybex (Cy) modelo Norm após 8 minutos no ciclo ergômetro. Foram feitas 3 repetições de reconhecimento e outras 5 para o teste, ambas a $60 \%$ s. Na análise estatística, foi usada a média de extensão máxima entre os 2 joelhos. A impulsão vertical (Iv) foi medida através de impulsímetro eletrônico, computando-se o melhor resultado de 3 tentativas. 
Foi aplicado o teste estatístico de Qui-quarado de Pearson aos resultados, onde cada variável foi dividida em 2 categorias: EM<217 e EM>217; Iv<59 e Iv>59, baseadas na média do grupo, rejeitando-se a hipótese nula a um nível de significância de 5\% (p<0.05) Resultados: Dos 23 atletas com EM $<217$, $73,9 \%$ tiveram Iv $<59$, assim como, dos 21 atletas com EM>217, 57,1\% tiveram Iv>59 ( $\mathrm{p}=0.04)$. Conclusão: Os resultados encontrados apontam relação significativa entre teste de Iv com o teste de força muscular isocinética de extensão média dos joelhos. Concluímos que o desempenho em Iv poderá estimar faixas de valores para a força máxima de extensão de joelhos. Contudo, são necessários estudos complementares com maiores populações.

\section{FORÇA MUSCULAR ISOMÉTRICA E ISOCI- NÉTICA DE EXTENSÃO DE PERNAS EM MENI- NOS E MENINAS NOS DIFERENTES GRAUS DE MATURIDADE}

Patrícia Schneider, Luciana Rodrigues, Andréa Fontoura, Flávia Meyer

LAPEX, Escola de Educação Física, UFRGS

Fundamentos: Meninos tendem a ser mais fortes do que as meninas, mas a diferença parece se acentuar a partir da puberdade. Objetivo: Comparar a força muscular isométrica e isocinética de membros inferiores entre meninos e meninas nos diferentes graus de maturidade. Metodologia: Um total de 57 voluntários saudáveis e não-atletas, 11 meninos e 10 meninas pré-púberes (PRÉ), 7 meninos e 9 meninas púberes (PUB), e 10 meninos e 10 meninas pós-púberes (PÓS) foram avaliados em um dinamômetro computadorizado (CYBEX). Foi medida a força isométrica nos ângulos 45 e $60^{\circ}$, e a força isocinética nas velocidades 60 e $90 \%$, da extensão de pernas. Resultados: A tabela abaixo mostra a média \pm dp da força isométrica e isocinética $(\mathrm{Nm})$ por grupo:

\begin{tabular}{|c|c|c|c|c|c|}
\hline & & Isométrico $45^{\circ}$ & Isométrico $60^{\circ}$ & $\begin{array}{l}\text { Isocinético } \\
60^{\circ} / \mathrm{s} \\
\end{array}$ & $\begin{array}{r}\text { socinético } \\
90 \% \mathrm{~s}\end{array}$ \\
\hline \multirow[t]{2}{*}{ PRÉ } & MENINO & $68,6 \pm 23,5$ & $83,2 \pm 22,9$ & $50,1 \pm 17,1$ & $44,7 \pm 14,8$ \\
\hline & MENINA & $53,9 \pm 11,7$ & $68,3 \pm 15,7$ & $40,6 \pm 9,5$ & $38,2 \pm 10,4$ \\
\hline \multirow[t]{2}{*}{ PUB } & MENINO & $119,0 \pm 23,3$ & $143,6 \pm 19,0$ & $87,1 \pm 24,2$ & $88,0 \pm 27,2$ \\
\hline & MENINA & $87,5 \pm 24,0 *$ & $103,9 \pm 21,7 *$ & $63,3 \pm 14,7 *$ & $64,5 \pm 14,2$ \\
\hline \multirow[t]{2}{*}{ PÓS } & MENINO & $167,7 \pm 31,4$ & $198,5 \pm 39,1$ & $136,4 \pm 37,0$ & $133,4 \pm 32,5$ \\
\hline & MENINA & $134,1 \pm 33,3^{*}$ & $155,7 \pm 41,5^{*}$ & $88,6 \pm 31,0^{*}$ & $90,0 \pm 23,4 *$ \\
\hline
\end{tabular}

Conclusão: Com exceção da força isocinética de $90 \%$, a força de extensão dos joelhos foi maior em meninos do que em meninas a partir da puberdade. (CNPq-UFRGS

\section{POTÊNCIA MUSCULAR NA FLEXÃO DE CO- TOVELO UNI E BILATERAL}

Roberto F. Simão Jr., Walace D. Monteiro, Claudio Gil S. de Araújo. Programa de Pós-Graduação em Educ. Física da Universidade Gama Filho, Laboratório de Fisiologia do Exercício/NUICAF - Aeronáutica e Clínica de Medicina do Exercício - CLINIMEX, Rio de Janeiro, RJ.

Fundamentação: exercícios de fortalecimento muscular podem ser realizados de forma uni e bilateral, contudo, pouco é sabido sobre a potência muscular (PM) e a carga máxima (CM) nessas condições. Objetivo: Comparar a PM e a CM na flexão do cotovelo entre os dois braços e entre a soma desses dois resultados com aquele obtido simultaneamente pelos dois braços. Metodologia: Submetemos 24 adultos jovens (14 homens) (PAR-Q negativo) e inexperientes no exercício de flexão de cotovelo ao teste de $1 \mathrm{RM}$ - duas repetições em velocidade máxima na fase concêntrica com $3 \mathrm{~s}$ de intervalo para cada carga -, com medida da potência (Fitrodyne, Bratislava), em cada braço e nos dois simultaneamente, em ordem randômica, para determinar a PM e a CM. Resultados: Os resultados para braço esquerdo e direito na $\mathrm{CM}-29,3 \pm 2,8$ e $29,7 \pm 2,9 \mathrm{~kg}$ - e na PM - $106 \pm 14$ e $109 \pm 12 \mathrm{~W}$ - foram similares $(p>0,05)$ e fortemente associados $(p>0,94)$. Comparando a soma dos valores unilaterais com os da execução bilateral, a CM era 5\% maior $(\mathrm{p}=0,02)$ e a PM 5\% menor ( $\mathrm{p}$ = 0,053). Conclusão: Apesar de todos serem destros, não houve diferenças unilaterais em CM e PM, provavelmente devido à inexperiência nesse exercício. A soma dos resultados unilaterais difere em $5 \%$ daquele obtido bilateralmente, mostrando, contudo, tendências opostas entre PM e CM, provavelmente refletindo uma limitação central na coordenação motora de um movimento complexo feito em máxima velocidade e com carga relativamente alta.

\section{RELAÇÃO DE EQUILÍBRIO ISOCINÉTICO AGONISTA/ANTAGONISTA: ESPECIFICIDADE NO ESPORTE}

Terreri, A S A P; Greve, J M D

Laboratório de Estudos do Movimento. Inst. de Ortopedia e Traumatologia Hosp.das Clínicas - Fac. Med. USP

Introdução: a dinamometria isocinética é um recurso valioso para o atleta, seja na sua avaliação funcional ou também na sua reabilitação frente a uma lesão do aparelho locomotor. As avaliações permitem a determinação do adequado equilíbrio muscular. Objetivo: caracterizar a proporção do equilíbrio muscular nos eversores e inversores do tornozelo entre atletas e não atletas. Metodologia: avaliação da eversão/inversão do tornozelo com dinamômetro isocinético em grupos de indivíduos assintomáticos não atletas e atletas de duas modalidades esportivas diferentes (corrida de curta distância e futebol de campo profissional). Velocidade angular de $30 \%$ seg. Resultados: notou-se que houve proporções de equilíbrio que diferiram entre si numa mesma velocidade angular isocinética. Observou-se que os atletas apresentaram força maior nos eversores comparados aos não atletas.Quanto aos atletas, os jogadores de futebol apresentaram, inclusive força maior de forma absoluta nos eversores no lado não dominante. Conclusões: pode-se considerar em alguns casos, que a atividade física pode ser um fator que venha a alterar a proporção agonista/antagonista de uma articulação. Cautela deve haver na decisão de se alterar tais proporções de equilíbrio no treinamento ou mesmo na orientação cinesioterápica. A conclusão de 
que uma articulação apresenta equilíbrio ou desequilíbrio muscular deve ser questionada e a atividade física deve ser considerada.

\section{ACURÁCIA DA EQUAÇÃO PREDITIVA DO V̇O $_{\text {2MÁx }}$ A PARTIR DE MEDIDAS ANTROPOMÉ- TRICAS}

Giovane G. Tortelote, Rodrigo L. Ferreira, Thiago L. Carvalho, Fernando A. M. S Pompeu

LABOFISE-UFRJ, Rio de Janeiro, RJ

Fundamentos: Equações para predição de $\dot{V}_{2 \mathrm{MÁx}}$ a partir de dados antropométricos são amplamente empregadas para orientar os testes de esforço. Entretanto tais equações são de precisão questionável na medida em que foram desenvolvidas para uma população com características distintas da brasileira. Objetivo: Verificar a relação entre o consumo máximo de oxigênio e sua predição por meio das variáveis peso, idade e estatura. Metodologia: A amostra foi composta por 7 homens e 2 mulheres com $22 \pm 2$ anos; $175 \pm 11 \mathrm{~cm} ; 72,0$ $\pm 13,0 \mathrm{~kg}$ (balança mecânica com estadiômetro - Whey ${ }^{\circledR}$, USA). Utilizou-se a metodologia sugerida por Wasserman et alii (1988) para os testes máximos em cicloergômetro mecânico $\left(\right.$ Monark $^{\circledR}$ Ind. Br) sendo realizada a análise de gases em circuito aberto (Aerosport ${ }^{\circledR}$, TEEM 100,USA) com medidas integradas a cada $20 \mathrm{~s}$. Para análise estatística utilizou-se o teste $t$ de student, coeficiente de correlação de Pearson e análise de regressão, aceitando-se o nível de significância de $p \leq 0,05$. Resultados: Não foi observada diferença significativa entre as médias dos valores medidos $\left(3,21 \pm 1,26 \mathrm{~L} \cdot \mathrm{min}^{-1}\right)$ e preditos $\left(2,94 \pm 0,71 \mathrm{~L} \cdot \mathrm{min}^{-1}\right), r=0,86$ e erro padrão da estimativa de $0,387 \mathrm{~L} \cdot \mathrm{min}^{-1}$. Conclusão: Existe relação positiva entre o $\dot{\mathrm{V}}_{2 \mathrm{MÁ}}$ e as variáveis antropométricas. No entanto, o alto valor do $E P E$ sugere a necessidade de desenvolvimento de um método mais acurado para população brasileira. Apoio: AACES-HSE

\section{EFEITOS DO AUMENTO DA CAPACIDADE AERÓBICA NA QUALIDADE DE VIDA E AUTO- NOMIA DOS IDOSOS}

Fatima S. Amorim, Estélio H.M. Dantas, Jani C.B. Aragão

PROCIMH, Universidade Castelo Branco, RJ

Fundamentos: Parte do declínio da vida autônoma pode ser atribuído a diminuição da atividade física. (BOILEAU \& col., 1999), afirmam que a atividade física regular está associada à manutenção e melhora da saúde física, capacidade funcional, tempo de vida e ao bem estar geral. Objetivos: O presente estudo tem a finalidade de investigar os efeitos do aumento da capacidade aeróbica na qualidade de vida e autonomia funcional em idosos. Metodologia: A pesquisa possui características de estudo experimental. A amostra utilizada para análise foi composta por 60 gerontes sedentários, com idade variando entre 60 a 70 anos. Na mensuração da variável independente utilizou-se o Canadian Aerobic Fitness test (POLLOCK \& WILMORE,1993). Para a avaliação do desempenho da autonomia funcional foi utilizado o Timed Up \& Go Test (PODSIADLO \& RICHARDSON, 1991) e para a autonomia nas atividades da vida diária foi aplicado um questionário de autoavaliação (POSNER,1995).Foram ainda coletados os dados através de um questionário de avaliação da qualidade de vida, protocolo de WHOQOL BREF,2000. Resultados: Houve melhoras significativas, (para $\mathrm{p}=0,05)$ na capacidade aeróbica, na qualidade de vida e autonomia dos idosos. Conclusão: Ocorreu melhoria na qualidade de vida e no grau de autonomia dos idosos na realização das habilidades funcionais selecionadas em decorrência do aumento da capacidade aeróbica, fruto de um programa de condicionamento físico.

\section{COMPARAÇÃO ENTRE AS MODALIDADES DE CAMINHADA E CORRIDA NA PREDIÇÃOO DO VंO}

Luiz Felipe L. Furtado, Bruno V. Montenegro, Tony M. dos Santos Laboratório de Fisiologia do Exercício - FISIOLAB - Universidade Estácio de Sá Rio de Janeiro, $R J$

Fundamentos: Apesar da literatura reportar a possibilidade da superestimação do $\mathrm{VO}_{2 \text { máx }}$ em virtude da utilização de protocolos inadequados, vários estudos comparativos entre protocolos de corrida foram feitos, mas poucos estudos compararam os protocolos de caminhada com o de corrida. Objetivo: Comparar o $\dot{\mathrm{VO}}_{2 \text { máx }}$, determinado pelos protocolos escalonados nas modalidades de caminhada e corrida na esteira rolante. Metodologia: Foram voluntários vinte e três indivíduos aparentemente saudáveis, sendo quinze homens, $(27,1 \pm$ 7,9 anos e $15,2 \pm 5,5 \%$ de gordura) e oito mulheres, $(28,9 \pm$ 7,6 anos e $26,9 \pm 8,3 \%$ de gordura). Na primeira visita ao laboratório, após a assinatura de um termo de consentimento, os indivíduos foram submetidos a uma avaliação antropométrica e teste de esforço máximo, de caminhada ou corrida, determinados aleatoriamente. Na segunda visita, num prazo não superior a uma semana, os voluntários realizaram a outra modalidade de teste máximo. Os dois protocolos foram equacionados para uma mesma intensidade em METs por estágio. O $\dot{\mathrm{VO}}_{2 \text { máx }}$ foi calculado com base nas equações sugeridas pelo ACSM (2000) utilizando-se o último estágio completado de cada protocolo. Resultados: Uma Anova a dois caminhos (tipo de atividade e sexo) não demonstrou diferenças significativas $(\mathrm{p}<0,05)$ entre o protocolo de caminhada $(42,4 \pm 6,2 \mathrm{ml} . \mathrm{kg}$ $\left.{ }^{1} \cdot \mathrm{min}^{-1}\right)$ e o de corrida $\left(43,4 \pm 5,5 \mathrm{ml} \cdot \mathrm{kg}^{1} \cdot \mathrm{min}^{-1}\right)$. Conclusão: A modalidade escolhida de teste parece não influenciar no resultado do $\dot{\mathrm{VO}}_{2 \text { máx }}$.

55 COMPORTAMENTO DA FREQÜÊNCIA CARDÍACA NA UTILIZAÇÃO DO TREINAMENTO DE FORÇA EM PACIENTES ISQUÊMICOS

Vivian Liane M. Pinto, Adriana L. Rozentul, Luísa R. de Meirelles, Simone A. Dino.

Hospital Universitário Pedro Ernesto - UERJ

Objetivo: Comparar o comportamento da freqüência cardíaca (FC) nas sessões de treinamento com e sem exercícios de 
força em pacientes isquêmicos que fazem parte do Núcleo de Prevenção e Reabilitação Cardíaca. Metodologia: Foram selecionados 17 pacientes isquêmicos, sendo 15 do sexo masculino e 2 do sexo feminino, com idade variando de 49 a 70 anos (média $=63,3)$. O período de observação foi de 15 a 16 sessões de treinamento, sendo monitorada a FC destes pacientes. Foi comparado o comportamento da FC nas sessões de treinamento aeróbio, dinâmico, envolvendo grandes grupamentos musculares, com sessões de treinamento com pesos para membros superiores que variaram entre 1 e $2 \mathrm{~kg}$. Resultados: A resposta da FC foi significativamente mais baixa, em todas as sessões de treinamento, quando os pacientes realizaram os exercícios com peso. Conclusão: Essa menor FC, caracterizando uma menor demanda de oxigênio pelo miocárdio, é um fator que deve ser levado em conta no treinamento de pacientes isquêmicos. Esses conhecimentos associados aos importantes efeitos dos exercícios de força, tem levado à utilização cada vez maior desses exercícios em programas de reabilitação cardíaca.

56 PERFIL DO IMC (ÍNDICE DE MASSA CORPORAL) DE INDIVÍDUOS QUE ESTÃO INICIANDO A PRÁTICA DE ATIVIDADE FÍSICA EM ACADEMIAS NA ILHA DO GOVERNADOR

Rogério M. Duarte, Ana Beatriz M. de C. Monteiro, Luiz B. Monteiro, Silvana R. U. Lobato, José F. Filho.

$U C B$ - Universidade Castelo Branco - Rio de Janeiro - RJ - Brasil

Fundamentos: O perfil do IMC de uma determinada população indica saúde ou morbidade relacionada à constituição física. Considera-se, desde Quetelet, que o IMC é um indicador válido de expectativa de vida e, portanto, tem sido utilizado por vários estudos clínicos. Sabendo se há ou não um perfil de peso inadequado de uma determinada população inativa, fica mais fácil determinar um plano de prevenção para as patologias relacionadas à obesidade ou carências nutricionais. Objetivo: Identificar o perfil do IMC dos indivíduos que iniciam a prática de atividade física em academias da Ilha do Governador. Metodologia: Esta é uma pesquisa experimental quantitativa. Foram levantados os valores da massa corporal e estatura, e a partir dos resultados foram calculados os respectivos Índices de Massa Corporal. Utilizou-se estatística descritiva para a determinação da proporção de indivíduos classificando-os como abaixo, dentro e acima dos valores referenciais de IMC determinados por Bray (1987). A amostra foi constituída de 120 indivíduos, iniciantes de programas de exercícios em academias da Ilha do Governador. O grupo foi dividido por faixa etária de 19 até 54 anos, seguindo a orientação dos limites desejáveis do IMC. Resultados: Entre os indivíduos mais jovens pesquisados há um predomínio de valores ideais para o IMC. Os dados evidenciaram também que quanto mais idade tem o aluno iniciante maior é o predomínio do IMC em valores acima ou abaixo do ideal. Conclusão: Os dados corroboram com o fato de que quanto mais idade tem um indivíduo, maior deve ser a busca de atividades físicas e para um controle do peso corporal.

\section{CONSUMO DE OXIGÊNIO EM CARGAS SUB- MÁXIMAS EM QUADRA EM FUTEBOLISTAS ADOLESCENTES}

Maria.A.P. D. M. Kiss; Ronaldo Vilela; Rômulo C. M. Bertuzzi; Edson Degaki; Karin A. Matsushigue; Hé1io A. Moura; Marcelo Regazzini; Emilson Colantonio

LADESP CENESP USP - São Paulo - SP

Fundamentos: Poucos estudos tem realizado a comparação direta de valores de $\dot{\mathrm{V}} 0_{2}$ em cargas submáximas em quadra e laboratório, contudo toma-se importante o conhecimento do que ocorre nas várias situações de treino e de competição, hoje acessíveis a partir da telemetria de V02. Objetivo: Avaliar a variável aeróbia em quadra e em laboratório. Metodologia: Comparamos 15 futebolistas, juvenis, da Ponte Preta de Campinas. Protocolos: 1) em quadra: corrida de $20 \mathrm{~m}$ ida e volta conforme Lèger e Lambert, 1982; 2) no laboratório: cargas crescentes em velocidade na esteira, a cada minuto- medida direta de $\dot{\mathrm{V}} \mathrm{O}_{2}$, respiração a respiração, com o K4b2 (Cosmed, Itália), em ambas as situações. Calculamos os $\dot{\mathrm{V}} \mathrm{O}_{2}$ de laboratório para as mesmas velocidades de quadra, interpolando-se linearmente com os 2 dados mais próximos. A comparação dos dados foi feita através de teste I Student para dados correlacionados e a ANOVA. Resultados: Os $\dot{\mathrm{V}} 0_{2}$ de quadra foram significativamente maiores do que em laboratório, a partir de $10 \mathrm{~km} . \mathrm{h}^{-1}$ os valores de $\dot{\mathrm{V}} 0_{2}$ são maiores nos grupos que apresentavam maior $\dot{\mathrm{V}} 0_{2}$ max (quadra em 10km.h- ${ }^{1}: \mathrm{El}$ 50,2; E3 53, 9; 12,5 km. h- ${ }^{1}$ : 63,9; E3 7 1,1ml.kg-'min'-'. Conclusão: Houve aumento do $\dot{\mathrm{V} 02}$ para a mesma velocidade média em teste de ida/volta, o que dificulta as predições de gasto energético durante jogo. Os indivíduos de maior $\dot{\mathrm{V}} \mathrm{O}_{2 \max }$ apresentaram maiores valores de $\dot{\mathrm{V}} 0_{2}$ submáximos, indicando menor economia de corrida e não necessariamente melhor condição aeróbia.

\section{RELAÇÃO ENTRE VंO,MÁX, ECONOMIA DE MOVIMENTO E DESEMPENHO EM TRIATLE- TAS}

Eduardo R. da Silva, Jerry R., Marcelo F.S. Cardoso, Alvaro R. Oliveira, Adroaldo C.A. Gaya

Escola de Educação Física - UFRGS

Fundamentos: $\mathrm{O} \mathrm{V}_{2}$ máx e a economia de movimento (ECO) podem ser fortes preditores do sucesso em atividades esportivas de endurance como nas modalidades que compõem o triatlo. Objetivos: Quantificar o $\mathrm{VO}_{2}$ máx e ECO em triatletas elite (EL) e não elite (NEL) em ciclismo (CIC) e corrida (COR); verificar a capacidade destas variáveis em discriminar a performance dos dois grupos da amostra. Metodologia: VO ${ }_{2}$ máx e ECO de triatletas (10 EL e 17 NEL) foram medidos em CIC e COR usando-se um protocolo incremental de $25 \mathrm{w} / \mathrm{min}$ e $1 \mathrm{Km} / \mathrm{h} / \mathrm{min}$ respectivamente. A ECO foi avaliada 
com 3 estágios de 7 minutos com cargas de 250,275 e $300 \mathrm{w}$ (CIC) e 15,16 e $17 \mathrm{Km} / \mathrm{h}$ (os valores de ECO foram relativizados pelo $\mathrm{V}_{2}$ máx). Foi utilizado o teste ANOVA para comparação inter-grupos e o nível de significância adotado foi de $\mathrm{P}<0,05$; foi utilizada o teste STEPWISE para análise da função discriminante. Resultados: A diferença foi significativa nas seguintes variáveis: $\dot{\mathrm{VO}}_{2 \mathrm{CIC}}-0,04 ; \dot{\mathrm{V}}_{2 \mathrm{COR}^{-}}-0,007$; $\mathrm{ECO}_{\mathrm{COR} 15 \mathrm{~km} / \mathrm{h}}-0,001 ; \mathrm{ECO}_{\mathrm{COR} 16 \mathrm{~km} / \mathrm{h}}-0,002 ; \mathrm{ECO}_{\mathrm{COR} 17 \mathrm{~km} / \mathrm{h}}-0,02$. $\mathrm{Na} \mathrm{ECO}_{\mathrm{CIC}}$ não houve diferença significativa a nenhuma intensidade $(250 \mathrm{w}-0,06 ; 275 \mathrm{w}-0,07 ; 300 \mathrm{w}-0,1)$. ECO15 ${ }_{\mathrm{km} / \mathrm{h}}$ foi selecionada pelo método Wilk's Lambda $(0,002)$, podendo classificar corretamente $81 \%$ dos atletas em seus grupos originais. Conclusão: a não-significância de ECO em CIC decorre da legalidade do vácuo nesta modalidade, pois igualou todos os atletas que competem em tal condição. Na corrida a individualidade é preservada, o que pode vir a discriminar a performance dos grupos.

\section{CONTROLE DE ATLETAS JUVENIS DE NATA- ÇÃO EM SISTEMA DE TREINAMENTO DE SO- BRECARGA AERÓBICA}

Gomes, André Luiz Marques; Estélio H. M. Dantas; Godoy, Eric. PROCIMH, Universidade Castelo Branco, Rio de Janeiro, RJ.

Fundamentos: A estrutura de um treinamento para jovens atletas é o fator de grande preocupação para o desenvolver de sua carreira adulta. Objetivos: Examinar as respostas fisiológicas dos atletas juvenis em um sistema de treinamento da natação, conhecido como Endurance Training em sobrecarga. Metodologia: O estudo verificou o nível de lactacidemia sangüínea em 19 atletas do sexo masculino com idade de 16 anos, em um treino de 10 repetições de 100 metros no nado de crawl com intervalo de 1:1 em intensidade ligeiramente acima do limiar anaeróbico. Coletando-se amostras sangüíneas no $1^{\circ}, 3^{\circ}, 5^{\circ}, 7^{\circ}, 9^{\circ}, 11^{\circ}, 13^{\circ}, 15^{\circ}$ e $20^{\circ}$ minutos. Com uma maquina de analise eletro-foto-enzimática da Accusport (Alemanha). Resultados: Encontramos, após um teste concentração média da máxima de $9 \mathrm{mmol} / \mathrm{l} \mathrm{com}$ um desvio padrão de 1,21 (para $r=0,54$ ) e uma concentração final de $1,4 \mathrm{mmol} / 1$ com um desvio médio de 0,64 (para $r=0,29$ ). Conclusão: $O$ estudo conclui que este sistema de treinamento aeróbico, é auxiliado pelo sistema de treinamento anaeróbico, observouse valores altos lactacidemia sangüínea, diferente dos valores descritos pela literatura Maglisho (1999), Navaro (1992), Kokobun (1999). O sistema de treinamento necessita de um grande trabalho de recuperação ativa para os níveis de lactacidemia sangüínea se restabelecerem aos valores iniciais. Recomenda-se utilizar volumes maiores para cada tiro, evitando desta maneira a facilidade impedir alta intensidade dada ao atleta no decorrer do estimulo, ou trabalhar com intervalos menores de recuperação entre os tiros. Pesquisar o sistema Endurance training em sobrecargas nos diferentes estilos, não pesquisados.

\section{VALIDADE DA ESTIMATIVA DO Vं ${ }_{\text {OzMÁx }}$ PELO MÉTODO DE ASTRAND PARA CICLO ERGÔME- TRO}

Fernando A.M.S. Pompeu

LABOFISE, Dep. Biociências da Atividade Física, EEFD/UFRJ

Fundamentos: $\mathrm{O} \dot{\mathrm{V}}_{\mathrm{O} \text { máx }}$ vem sendo estimado a partir da relação da $\mathrm{FC} \mathrm{e} \dot{\mathrm{V}}_{\mathrm{O} 2}$ durante o esforço de intensidade leve a moderada. Objetivo: Comparar o $\dot{\mathrm{V}}_{\text {O2máx }}$ (platô do $\dot{\mathrm{V}}_{\mathrm{O} 2} \leq 0,20$ $\mathrm{L} \cdot \mathrm{min}^{-1}, \mathrm{RER} \geq 1,1,[\mathrm{Lac}] \geq 8 \mathrm{mmol} \cdot \mathrm{L}^{-1}, \mathrm{Bog} \geq 18$ e FC $\geq 85 \%$ $\left.\mathrm{FC}_{\text {max }}\right)$ com suas estimativas pela relação $\mathrm{FC} / \dot{\mathrm{V}}_{\mathrm{O} 2}\left(\mathrm{Est}_{\mathrm{O}} \dot{\mathrm{O}}_{\text {máx }}\right)$ e FC/waat $\left(\right.$ NomV $\left._{\text {O2max }}\right)$. Metodologia: Foram sujeitos deste estudo 15 indivíduos (5? e 10?) com $22 \pm 3$ anos, $74,3 \pm 14,4 \mathrm{~kg}$, $176 \pm 9 \mathrm{~cm}$ e $17,9 \pm 8,8 \%$ gordura relativa. Estes sujeitos foram submetidos à carga de $\approx 50 \% \dot{\mathrm{V}}_{\text {O2máx }}$ por 5 minutos e, após 10 minutos de recuperação passiva, à carga máxima por 2 a 3 min. (ciclo Monarch ${ }^{\circledR}, \mathrm{Br}$ ). A FC foi registrada pelo sistema telimétrico (Polar Electro, Finland). A espirometria foi realizada em circuito aberto (Aerosport ${ }^{\circledR}$ TEEM 100, USA) e a lactacidemia ([Lac]) medida pelo método eletroenzimático (YSI 1500 Sport, USA). A ANOVA com uma classificação e o teste post hoc de Tukey foram empregados para comparação dos métodos $(p \leq 0,05)$. Resultados: Não houve diferença significativa entre o $\dot{\mathrm{V}}_{\text {O2máx }}(2,74 \pm 0,97)$ e suas estimativas

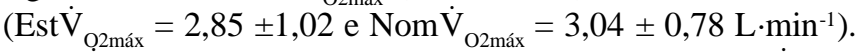
A EstV $\mathrm{O}_{\text {O2max }}$ apresentou $r=0,91(E P E=0,44)$ e a NomV $\dot{\text { O2máx }}$ de $r=0,85\left(E P E=0,50 \mathrm{~L} \cdot \mathrm{min}^{-1}\right)$ com o $\dot{\mathrm{V}}_{\mathrm{O} \text { máx }}$. Conclusão: $\mathrm{O}$ método para estimativa do $\dot{\mathrm{V}}_{\text {O2máx }}$ proposto por Saltin \& Astrand, (1967) apresenta satisfatória validade externa. Apoio: FUJB/UFRJ e AACEA-HSE/RJ.

\section{CORRELAÇÃO ENTRE DERMATOGLIFIA E APTIDÃO FÍSICA EM ATLETAS DE FUTSAL ADULTO MASCULINO}

Paulo M. S. Dantas, José F. Filho e André L. K. Castanhede.

Universidade Castelo Branco, RJ/RJ.

Fundamentos: A aptidão física do atleta de alto rendimento de futsal está ligada a sua performance. Faz-se necessário o conhecimento da modalidade por diversos ângulos, de funcionais a genéticos. Os índices dermatoglíficos sofrem alterações de acordo com os níveis de qualificação esportiva, (CUMMINS e MIDLO, 1942). Objetivo: Estabelecer uma correlação entre variável dermatoglifia e as variáveis $\mathrm{V}_{2 \max }$ /impulsão vertical, dos jogadores da equipe de futsal masculino adulto, do Vasco da Gama. Metodologia: A associação entre as variáveis foi investigada, através do coeficiente bivariado de Pearson, sempre com $\mathrm{a}=0,001$. A hipótese testada foi que o valor do coeficiente era significativo, na indicação da existência de associação entre as variáveis estudadas, assim estabelecemos: H0: r não é significativo; H1: r é significativo. A amostra total foi de 13 atletas, idade $=25.53 \pm 2.65$, da equipe de futsal campeão da Liga Nacional 2000. Testes utilizados: $\dot{\mathrm{V}} \mathrm{O}_{2 \text { máx. }}$ (Multistage Fitness Test, Loughborough University, 1987); Dermatoglifia (CUMMINS e MIDLO, 1942) e Impul- 
são Vertical _ (Jump Test, Johnson \& Nelson 1979). Resultados: $\dot{\mathrm{VO}}_{2 \max }$ : Coeficiente. _ $\mathrm{A}=0.962, \mathrm{~L}=0.831, \mathrm{~W}=0.932$, D10 $=0.975$ e SQTL $=0.962$; Significância $\_.000$ para todos; Imp. Vertical: Coeficiente _ A $=0.944, \mathrm{~L}=0.807, \mathrm{~W}=$ 0.834 , D10 $=0.889$ e SQTL $=0.944$, Significância $\_0.000$ para todos exceto $\mathrm{L}=0.001$. Conclusões: As análises dos testes nos levaram a rejeitar $\mathrm{H} 0$, demonstrando a existência de correlação entre as variáveis investigadas.

\section{ANÁLISE SOBRE O COMPORTAMENTO DA FREQÜÊNCIA CARDÍACA EM ATIVIDADES TERRESTRE E AQUÁTICA}

Helio L. Furtado, Fernanda B. Beltrão, José F. Filho Universidade Castelo Branco, RJ/RJ

Fundamentos: Pode-se esperar que o exercício físico aquático produza reações fisiológicas diferentes daquelas ao ar livre devido tanto ao efeito hidrostático da água nos sistemas cardiorrespiratórios como a sua capacidade de intensificar a perda de calor comparada ao ar, (AVELINE et al). Objetivo: Analisar a variação de freqüência cardíaca em atividades terrestres e aquáticas durante a realização de um teste de Cooper de 12 minutos de corrida em universitários do $7^{\circ}$ período de Educação física da UCB. Metodologia: A associação entre as variáveis foi investigada, através do teste do sinal com a = 0,05. A hipótese testada foi que o valor do coeficiente era significativo, na indicação de existência entre as variáveis estudadas, assim estabelecemos: H0: não há diferença nas freqüências cardíacas na pista e na água; H1: há diferença nas freqüências cardíacas na pista e na água. A amostra total foi de 18 alunos. Resultados: Nos 13 testes estatísticos realizados para cada indivíduo, 11 apresentaram diferença entre as freqüências em um nível de significância de 5\%. Conclusões: As análises dos testes levaram a rejeitar H0, demonstrando que há evidências estatísticas de que existe diferença significativa nas frequiências cardíacas entre o teste realizado em corrida na pista e o teste realizado em corrida na água.

\section{TRIATLETAS PROFISSIONAIS: FREQÜÊN- CIA CARDÍACA, CONCENTRAÇÕES DE LACTA- TO E ÍNDICE DE PERCEPÇÃO DE ESFORÇO DE BORG}

Emilson Colantonio; Roberto F. Costa; Ronaldo V. Barros; Maria Augusta P. D. Kiss.

CENESP / LADESP / EEFEUSP; UNIMONTE, SP; UNICID, SP.

Fundamentos: $\mathrm{O}$ triatlo é um esporte de endurance onde os atletas competem em Natação (Nat), Ciclismo (Cic) e Corrida (Cor), respectivamente, onde o componente determinante é a habilidade de sustentar uma taxa de dispêndio energético por períodos prolongados de tempo. A frequiência cardíaca (FC) é utilizada como parâmetro de referência na prescrição e controle do treinamento. O índice de percepção de esforço (IPE), correlaciona-se bem com diferentes variáveis de exercício. Objetivo: Verificar a relação entre os valores encontrados de 112
FC, [La] e IPE, em testes de campo com cargas progressivas para Nat, Cic e Cor. Metodologia: Amostra: 5 triatletas profissionais (idade $=22,80 \pm 3,96$; massa corporal $=74,60 \pm$ $1,67 \mathrm{~kg}$; estatura $=176,00 \pm 3,31 \mathrm{~cm})$. Os testes realizados foram 4 x 400m (Nat); 4 x 5000m (Cic) e 5 x 1000 (Cor), em dias alternados. Para as análises das [La] foi utilizado o Lactímetro Accusport ${ }^{\circledR}$, para a FC o Frequiencímetro Polar ProTrainer® e para o IEP a escala subjetiva de esforço original de 6 a 20 (BORG, 1982). A análise estatística foi realizada através da técnica de Spearman rho. Resultados: Grupo como um todo, as correlações significativas foram: Carga $1=$ IPE x [La] (0,622*); Carga 2 = IPE x [La] (0,696*); Carga 4 = IPE x [La] $\left(0,493^{*}\right)$. Por modalidade, as correlações significativas foram: Carga $1 \mathrm{Cic}=\mathrm{FC} \mathrm{x}[\mathrm{La}]\left(0,845^{*}\right)$; Carga $2 \mathrm{Cic}=\mathrm{IPE} \mathrm{x}$ [La] $\left(0,820^{*}\right)$; Carga 3 Nat $=$ FC x [La] $\left(0,880^{*}\right)$, Cic $=$ IPE x [La] $\left(0,882^{*}\right)$, Cor $=$ FC x [La] $\left(0,926^{* *}\right)$. Conclusão: A FC não se correlacionou com o IPE, apenas a análise da FC não é suficiente para o controle adequado das cargas em treinamento de triatletas de alto nível.

\section{MEDIÇÃO DA VELOCIDADE CORRESPON- DENTE AO LIMIAR ANAERÓBIO EM ATLETAS DE FUTEBOL PROFISSIONAL, UTILIZANDO UMA ÚNICA AMOSTRA DE LACTATO NO SAN- GUE}

Luiz A. Crescente e Félix A. Drummond

Sport Club Internacional, LAFIMED-ULBRA, C.I.M.E., RS.

Fundamentos: $\mathrm{O}$ surgimento de vários testes e protocolos de avaliação fisiológica foi uma consequiência da valorização da ciência no futebol. Objetivos: O presente estudo verifica a aplicação de um teste proposto por pesquisadores de Milão (ITA), em 1993, que utiliza-se do método metabólico para avaliar a velocidade correspondente ao limiar anaeróbio (LA) em atletas de futebol. Metodologia: O teste é realizado na pista ou no campo, consiste em correr durante seis minutos na velocidade constante de $13,5 \mathrm{Km} / \mathrm{h}$. Ao final coleta-se uma gota de sangue para determinar o nível de lactato. $\mathrm{O}$ valor encontrado é transposto para uma fórmula de predição, que corresponde a velocidade do LA em $\mathrm{Km} / \mathrm{h}$. Neste estudo foi utilizado para a análise do lactato sanguíneo o aparelho ACCUSPORT- Boehringer Mannheim e foram realizadas cinco coletas no período de Julho/2000 à Março/2001, com atletas de uma equipe de futebol profissional da $1^{\text {a }}$ divisão brasileira, cujos resultados médios apresentamos a seguir. Resultados:

\begin{tabular}{l|c|c|c}
\hline DATA & ATLETAS & LACTATO & VELOCIDADE \\
\hline & $\mathrm{N}^{\mathbf{0}}$ & $\mathrm{Mmol} / 1$ & $\mathrm{Km} / \mathrm{h}$ \\
\hline Jul/00 & 20 & 4.0 & 13.4 \\
\hline Set/00 & 25 & 4.6 & 13.1 \\
\hline Dez/00 & 25 & 5.3 & 12.7 \\
\hline Jan/01 & 20 & 6.5 & 12.0 \\
\hline Mar/01 & 30 & 4.7 & 13.0 \\
\hline
\end{tabular}

Rev Bras Med Esporte - Vol. 7, № 3 - Mai/J un, 2001 
Conclusões: Os resultados mostram que o método pode ser utilizado para a avaliação do LA em atletas de futebol. É de fácil aplicabilidade, de rápida execução e tem boa aceitação por parte dos atletas.

\section{COMPARATIVO ENTRE RECUPERAÇÃO ATI- VA E ATIVA CONTROLADA NO TREINAMENTO DE NATAÇÃO}

Erik Godoy; Estélio H. M. Dantas; André Luiz Marques Gomes. PROCIMH, Universidade Castelo Branco, Rio de Janeiro, RJ.

Fundamentos: A otimização da recuperação após treinamentos com intensidades de moderada a alta são aspectos importantes para o desenvolvimento ideal do atleta de natação. Objetivos: Examinar as respostas fisiológicas de atletas juvenis de sexo feminino durante a recuperação ativa e recuperação ativa controlada após a execução de uma série padronizada de 10 tiros de 100 metros. Metodologia: O estudo verificou o nível de lactacidemia sangüínea em 15 atletas de sexo feminino com idade de 16 anos, na recuperação ativa e ativa controlada após um treino de 10 repetições de 100 metros no nado de crawl com intervalo de 1:1 em intensidade ligeiramente acima do limiar anaeróbico, coletando-se amostras sangüíneas para análise eletro-foto-enzimática (Accusport - Alemanha) após o esforço para determinação da concentração máxima e após o trabalho regenerativo, $800 \mathrm{~m}$ (ativo) e 8x100m (ativo controlado). Resultados: Após determinação do $\mathrm{La}_{\text {máx. }}(\mathrm{x}=7,16 \mathrm{mmol} / \mathrm{l} \pm 0.8 ; \mathrm{r}=0,40)$ o trabalho regenerativo ativo apresentou concentração média de $5,39 \mathrm{mmol} / \mathrm{l} \mathrm{com}$ $\mathrm{DP}=0,94(\mathrm{r}=0,48)$ e o trabalho regenerativo ativo controlado 2,71 mmol/1 com DP = 0,83 ( $\mathrm{r}=0,29)$. Conclusão: $\mathrm{O}$ estudo conclui que a recuperação ativa controlada é significativamente mais rápida do que a recuperação ativa, conforme demonstrado pelos níveis de lactacidemia obtidos com cada trabalho regenerativo. Recomenda-se o emprego do controle de intensidade no trabalho regenerativo, para otimizar a recuperação dos estímulos de treinamento com intensidades ligeiramente superiores a do limiar anaeróbico. 